\title{
Structure and dynamics of water and lipid molecules in charged anionic DMPG lipid bilayer membranes
}

Klinge Rønnest, Andreas ; Peters, Günther H.J.; Hansen, Flemming Yssing; Taub, H.; Miskowiec, A.

Published in:

Journal of Chemical Physics

Link to article, DOI:

$10.1063 / 1.4945278$

Publication date:

2016

Document Version

Publisher's PDF, also known as Version of record

Link back to DTU Orbit

Citation (APA):

Klinge Rønnest, A., Peters, G. H. J., Hansen, F. Y., Taub, H., \& Miskowiec, A. (2016). Structure and dynamics of water and lipid molecules in charged anionic DMPG lipid bilayer membranes. Journal of Chemical Physics, 144(14), [144904]. https://doi.org/10.1063/1.4945278

\section{General rights}

Copyright and moral rights for the publications made accessible in the public portal are retained by the authors and/or other copyright owners and it is a condition of accessing publications that users recognise and abide by the legal requirements associated with these rights.

- Users may download and print one copy of any publication from the public portal for the purpose of private study or research.

- You may not further distribute the material or use it for any profit-making activity or commercial gain

- You may freely distribute the URL identifying the publication in the public portal 


\section{AlP | $\begin{aligned} & \text { The Journal of } \\ & \text { Chemical Physics }\end{aligned}$}

\section{Structure and dynamics of water and lipid molecules in charged anionic DMPG lipid bilayer membranes}

A. K. Rønnest, G. H. Peters, F. Y. Hansen, H. Taub, and A. Miskowiec

Citation: The Journal of Chemical Physics 144, 144904 (2016); doi: 10.1063/1.4945278

View online: http://dx.doi.org/10.1063/1.4945278

View Table of Contents: http://scitation.aip.org/content/aip/journal/jcp/144/14?ver=pdfcov

Published by the AIP Publishing

\section{Articles you may be interested in}

Molecular dynamics study of lipid bilayers modeling the plasma membranes of mouse hepatocytes and hepatomas

J. Chem. Phys. 144, 085104 (2016); 10.1063/1.4942159

Diffusion and spectroscopy of water and lipids in fully hydrated dimyristoylphosphatidylcholine bilayer membranes

J. Chem. Phys. 140, 104901 (2014); 10.1063/1.4867385

A molecular dynamics study on heat conduction characteristics in DPPC lipid bilayer

J. Chem. Phys. 133, 154705 (2010); 10.1063/1.3481650

Stretching effects on the permeability of water molecules across a lipid bilayer

J. Chem. Phys. 127, 105104 (2007); 10.1063/1.2764079

Molecular dynamics study of a lipid bilayer: Convergence, structure, and long-time dynamics

J. Chem. Phys. 106, 5731 (1997); 10.1063/1.473592

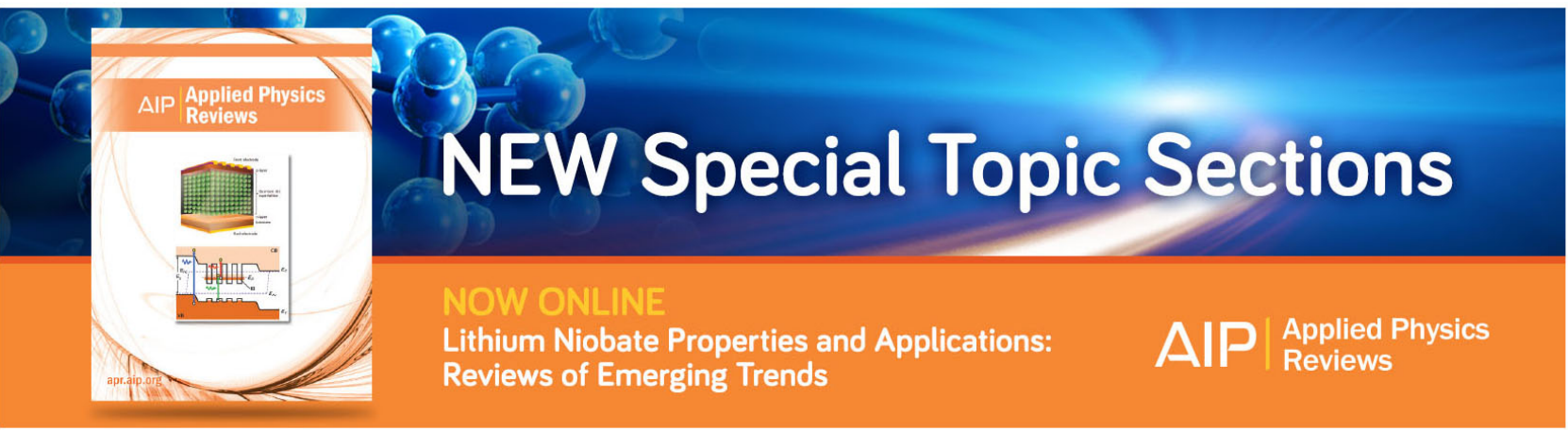




\title{
Structure and dynamics of water and lipid molecules in charged anionic DMPG lipid bilayer membranes
}

\author{
A. K. Rønnest, ${ }^{1}$ G. H. Peters,${ }^{1}$ F. Y. Hansen, ${ }^{1, a)}$ H. Taub, ${ }^{2}$ and A. Miskowiec ${ }^{2}$ \\ ${ }^{1}$ Department of Chemistry, Technical University of Denmark, IK 207 DTU, DK-2800 Lyngby, Denmark \\ ${ }^{2}$ Department of Physics and Astronomy and the University of Missouri Research Reactor, \\ University of Missouri, Columbia, Missouri 65211, USA
}

(Received 15 October 2015; accepted 21 March 2016; published online 14 April 2016)

\begin{abstract}
Molecular dynamics simulations have been used to investigate the influence of the valency of counter-ions on the structure of freestanding bilayer membranes of the anionic 1,2-dimyristoyl-snglycero-3-phosphoglycerol (DMPG) lipid at $310 \mathrm{~K}$ and $1 \mathrm{~atm}$. At this temperature, the membrane is in the fluid phase with a monovalent counter-ion and in the gel phase with a divalent counter-ion. The diffusion constant of water as a function of its depth in the membrane has been determined from mean-square-displacement calculations. Also, calculated incoherent quasielastic neutron scattering functions have been compared to experimental results and used to determine an average diffusion constant for all water molecules in the system. On extrapolating the diffusion constants inferred experimentally to a temperature of $310 \mathrm{~K}$, reasonable agreement with the simulations is obtained. However, the experiments do not have the sensitivity to confirm the diffusion of a small component of water bound to the lipids as found in the simulations. In addition, the orientation of the dipole moment of the water molecules has been determined as a function of their depth in the membrane. Previous indirect estimates of the electrostatic potential within phospholipid membranes imply an enormous electric field of $10^{8}-10^{9} \mathrm{~V} \mathrm{~m}^{-1}$, which is likely to have great significance in controlling the conformation of translocating membrane proteins and in the transfer of ions and molecules across the membrane. We have calculated the membrane potential for DMPG bilayers and found $\sim 1 \mathrm{~V}$ $\left(\sim 2 \cdot 10^{8} \mathrm{~V} \mathrm{~m}^{-1}\right)$ when in the fluid phase with a monovalent counter-ion and $\sim 1.4 \mathrm{~V}\left(\sim 2.8 \cdot 10^{8}\right.$ $\mathrm{V} \mathrm{m}^{-1}$ ) when in the gel phase with a divalent counter-ion. The number of water molecules for a fully hydrated DMPG membrane has been estimated to be 9.7 molecules per lipid in the gel phase and 17.5 molecules in the fluid phase, considerably smaller than inferred experimentally for 1,2-dimyristoyl-sn-glycero-3-phosphorylcholine (DMPC) membranes but comparable to the number inferred for 1,2-dilauroyl-sn-glycero-3-phosphoethanolamine (DLPE) membranes. Some of the properties of the DMPG membrane are compared with those of the neutral zwitterionic DMPC bilayer membrane at $303 \mathrm{~K}$ and $1 \mathrm{~atm}$, which is the same reduced temperature with respect to the gel-to-fluid transition temperature as $310 \mathrm{~K}$ is for the DMPG bilayer membrane. (C) 2016 AIP Publishing LLC. [http://dx.doi.org/10.1063/1.4945278]
\end{abstract}

\section{INTRODUCTION}

Over the last couple of decades, there has been a great interest in studying structural and dynamical properties of phospholipid bilayers as models for the more complex biological cell membranes. The motivation for these studies has been to obtain an understanding of the functioning of cell membranes as a barrier between the interior and exterior of the cell and as a matrix for integral transmembrane proteins, which enable transport of ions and molecules through the membrane.

A number of different experimental techniques like $\mathrm{NMR},{ }^{1-3}$ time-resolved fluorescence spectroscopy, ${ }^{4}$ neutron scattering, ${ }^{5-8,11,18}$ ultrafast spectroscopy, ${ }^{9}$ and IR absorption spectroscopy ${ }^{10}$ have been used to study hydration water dynamics at the interface between a lipid bilayer membrane and bulk water. Most of these studies have been conducted

\footnotetext{
a) Author to whom correspondence should be addressed. Electronic mail: flemming@kemi.dtu.dk
}

on stacks of bilayer membranes to give a sufficiently intense signal. However, it is difficult to interpret the neutron scattering spectra of these multilayer membrane stacks because of the possible interactions between the membranes as well as the unknown amount of water between them. ${ }^{11,18}$

With the computational power available today, molecular dynamics (MD) and Monte Carlo simulations play an increasing role in structural and dynamical studies of lipid bilayers ${ }^{13-16}$ because it is now feasible to do all-atom simulations of the lipid systems on time scales similar to those accessible in NMR and neutron scattering experiments. ${ }^{17}$ Our simulation results are compared with quasielastic neutron scattering results on a single-supported 1,2-dimyristoylsn-glycero-3-phosphoglycerol (DMPG) bilayer system, so possible complications in the interpretation of results from multilayer systems are avoided. ${ }^{11,18}$

There are a variety of lipids with different head groups and alkane chains. Much work has been devoted to the study of phosphatidylcholines (PCs), the major 
constituent of mammalian plasma membranes. They have been studied in the biologically most relevant state of the biomembrane, the "fluid" or liquid-crystal phase. We have previously done MD simulations of the 1,2-dimyristoyl-snglycero-3-phosphorylcholine (DMPC) system, investigating the structure and dynamics of both water and lipid molecules ${ }^{17}$ and report here the results of MD simulations on a freestanding DMPG lipid bilayer. DMPG is also an important constituent of biological membranes but less abundant than the PC lipids. The two lipids have identical aliphatic chains but different head groups in that the choline subunit in DMPC has been replaced with a glycerol group in DMPG. Another important difference is that the DMPG lipid is charged at the phosphate group in contrast to DMPC, which is a neutral zwitterion, so that the properties of the DMPG bilayer may also depend on the counter-ion. The two lipids are sketched in Fig. 1 to show their chemical composition; the molecules are very flexible with many conformations that may differ from those in the sketch. In (c), we have shown the average orientation of the head group with respect to the surface of the membrane as determined from the simulations. It is found that the polar angle of the vector from the phosphate atom in the DMPG headgroup to the central glycerol carbon atom in the headgroup is $\sim 80^{\circ}$ with

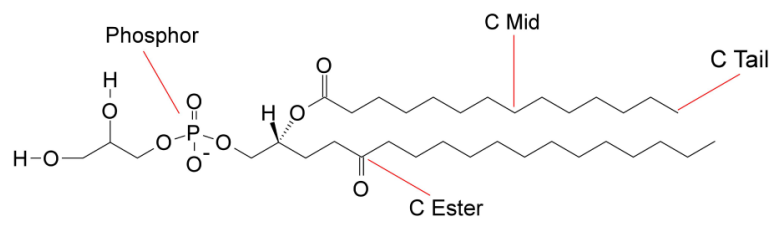

(a)
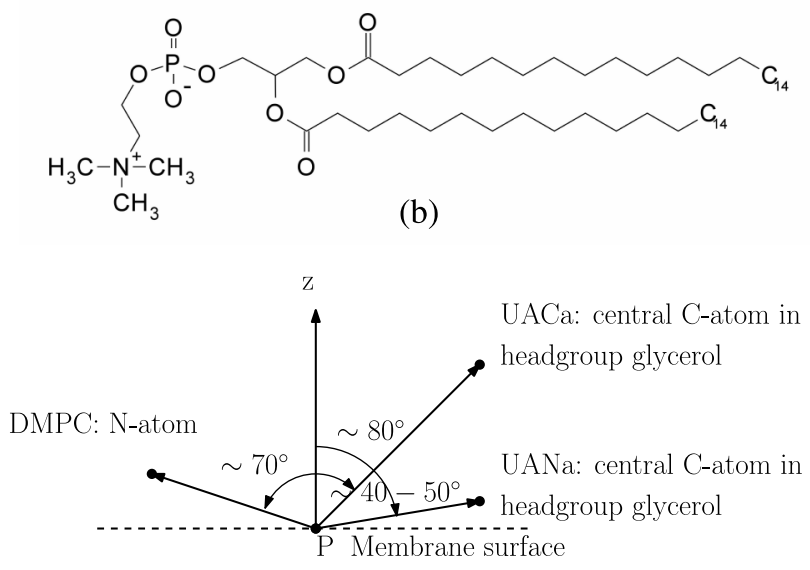

(c)

FIG. 1. (a) Diagram of a DMPG lipid molecule with an identification of the selected atoms in the lipid to be considered and (b) of a DMPC lipid molecule for comparison. To the glycerol backbone are attached two 14 $\mathrm{C}$-atom aliphatic chains and a phosphate group, which connect via an ethyl bridge to a glycerol group in DMPG and to a choline group in DMPC. The former is thus a charged anionic molecule with a counter-ion to form a neutral salt, while the latter is a neutral zwitterionic molecule. Note that these are only diagrams of the molecules, which show the chemical composition and not one of many conformations. (c) Diagram of the orientation of the head group with respect to the aliphatic chains along the $z$-direction perpendicular to the membrane surface. The orientation of the DMPG head group is given by the polar angle of the vector connecting the phosphorous atom and central carbon atom in the glycerol unit in the headgroup. Also shown for comparison is the orientation of the head group in the DMPC bilayer membrane given by the polar angle of the vector connecting the phosphorous and nitrogen atoms. sodium as the counter-ion, which means that the head group is almost parallel to the membrane surface. The polar angle is somewhat smaller, $\sim 40^{\circ}-50^{\circ}$, with calcium as the counter-ion probably due to the closer packing of the lipid molecules in the gel phase. For DMPC, the polar angle of the head group, defined by the vector from the phosphate atom to the nitrogen atom, is $\sim 70^{\circ}$.

In our MD simulations, we have analyzed the effect of monovalent and divalent counter-ions on the structure of the bilayer DMPG membrane and found that, at the given temperature of $310 \mathrm{~K}$, a change from a monovalent counterion to a divalent one causes the membrane to transform from a fluid to a gel phase. Said differently, the gel-to-fluid transition temperature is higher with a divalent counter-ion than with a monovalent one. The driving force for that transition seems to be the higher affinity of the calcium ion to the phosphate group in the lipid and its capability to bind simultaneously to two lipids, which is not seen with a monovalent counter-ion.

This phase change is also manifested in the diffusion constant of water molecules as a function of their depth in the membrane as well as in the center-of-mass diffusion constant for the lipid molecules. The diffusion constants have been determined by mean-square-displacement calculations and, for the water molecules, also by calculation of the incoherent quasielastic neutron scattering functions. The latter are compared to experimental quasielastic neutron scattering results. The orientation of the water molecule dipoles as a function of their depth in the membrane has been analyzed and shows significant differences in membranes with monovalent and divalent counter-ions. We also find that some water molecules are more strongly bound to the phosphorous atom in the head groups, when the counter-ion is divalent calcium rather than monovalent sodium.

The electrostatic potential in the phospholipid membranes is of importance for the conformation of translocating proteins and the transport of ions and molecules across the membrane. It cannot be measured directly but has been estimated by indirect methods. For the PC lipid membranes, the potentials have been estimated to be in the range of $200-400 \mathrm{mV}^{19}$ whereas there appear to be no estimates for PG bilayer membranes. We have therefore used our MD simulation results to calculate the potential using the Poisson equation and find potentials of $\sim 1 \mathrm{~V}$ and $\sim 1.4 \mathrm{~V}$ for membranes with monovalent and divalent counter-ions, respectively.

The paper is organized in 9 sections. Section II has details about the systems, simulations, and force field. Section III describes the effect of the counter-ion on the structure of the membrane. Section IV follows with a determination of the atomic density profiles in the direction perpendicular to the membrane surface. Section V has a mean-square-displacement calculation of the diffusion constant of water molecules as function of their depth in the membrane. The center-of-mass diffusion constant for the lipid molecules is calculated as well. For the water molecules, we have also calculated the incoherent quasielastic neutron scattering functions, which are compared with observed spectra. In Section VI, our simulations are compared with the results of neutron scattering measurements. In Section VII, the strength of the binding of water molecules to the phosphorous atom in the head groups is 
determined. Section VIII has a determination of the orientation of the water molecule dipole moments as a function of their depth in the membrane. In Section IX, we describe the results of the calculation of the electrostatic potential in the membrane. Section X has our conclusions.

\section{MD SIMULATION}

\section{A. System}

The system in the simulations consists of a single freestanding bilayer with 64 DMPG molecules per leaflet (a total of 128 lipid molecules), 6760 water molecules distributed evenly in two water layers on the membrane surfaces, and 128 sodium or 64 calcium counter-ions. That gives 52 water molecules per lipid molecule well above the amount required for full hydration. For DMPC membranes, that number has been given as $\sim 27$ molecules per lipid, ${ }^{20}$ while for DLPE membranes, the number is significantly smaller, $\sim 10$ molecules. ${ }^{21,22}$ We have not been able to find numbers for DMPG membranes and have therefore made an estimate from the simulations. We find $\sim 9.7$ water molecules per lipid for the membrane in the gel phase and $\sim 17.5$ molecules in the fluid phase as described in Section IV. The thickness of the water layers on either side of the membrane is $\sim 25 \AA$ in the simulations with the sodium counter-ion and $\sim 30 \AA$ with the calcium counter-ion. We have also done a simulation with a $0.1 \mathrm{M}$ sodium chloride salt solution to see the effect of an increasing ionic strength.

Because we will compare the DMPG simulation results with the ones for the DMPC system, ${ }^{17}$ the simulations were done at identical reduced temperatures relative to their gelto-fluid transition temperature. Aqueous dispersions ${ }^{23}$ of the anionic phospholipid DMPG in $100 \mathrm{mM}$ ionic strength at $\mathrm{pH}$ values higher than 4 are known to exhibit a thermal behavior rather similar to that of the zwitterionic DMPC lipid bilayer, which undergoes a gel-to-fluid transition at $23{ }^{\circ} \mathrm{C}$. However, in aqueous media of low ionic strength, DMPG has been shown to present a large gel-to-fluid transition region ranging from 18 to $35^{\circ} \mathrm{C}$. This intermediate phase is characterized by a continuous change in membrane packing. At a lipid concentration of $\sim 800-900 \mathrm{mM}$ and higher and at low ionic strength, the region with the intermediate phase seems to disappear, and there is a single gel-to-fluid transition temperature of $25-28{ }^{\circ} \mathrm{C}$. These conditions are quite similar to those found in the simulations with an ionic strength of zero (except for the simulations with a $0.1 \mathrm{M} \mathrm{NaCl}$ salt concentration) and a lipid concentration of $\sim 0.6 \mathrm{M}$ with the monovalent sodium counter-ion.

\section{B. Simulations}

The simulations were done with the NAMD $2.8 \operatorname{program}^{24}$ using a time step of 1 fs and saving coordinates every $0.5 \mathrm{ps}$, corresponding to a dynamical range of $4.14 \mathrm{meV}$ in the calculation of the quasielastic neutron scattering functions. The simulations were run in time-blocks of 4-5 ns and done in the NPT ensemble with the temperature controlled by a Langevin thermostat with a damping coefficient of $5 \mathrm{ps}^{-1}$.
Pressure was controlled by a Nosé-Hoover Langevin barostat with a piston oscillation time of 100 fs and a damping time of $50 \mathrm{ps}$. The long-range electrostatic interactions were calculated using the particle mesh Ewald method with a $\sim 1 \AA$ grid size. The electrostatic interactions were updated every fourth femtosecond. van der Waals interactions were cut off at $12 \AA$ in combination with a switching function starting at $10 \AA$. Periodic boundary conditions were used in all three Cartesian directions. Some earlier reported simulations ${ }^{25}$ had the ratio between the box lengths in the $x$ - and $y$-directions fixed, but we found no significant changes in the results when there were no constraints on the box lengths. So, in our simulations, they are free to change independently as determined by the barostat. The box dimensions fluctuate during the simulations and are approximately $65 \AA$ in the $x$-direction, $65 \AA$ in the $y$-direction, and $90 \AA$ in the $z$-direction in the simulations with sodium as the counter-ion and $55 \AA, 55 \AA$, and $110 \AA$, respectively, in the simulations with the calcium ion.

\section{Force field}

We tested different force fields and used a comparison of the area/lipid in the simulation with experimental values in order to choose the optimal force field. The water was modeled by the flexible 3-point TIP3P potential, which is known to overestimate the dielectric constant and dipole moment ${ }^{26}$ but gives diffusion constants in good agreement with experiments. The simulations were started from a non-equilibrated system with an area per lipid-molecule of about $70 \AA^{2}$. We have shown the area/lipid as a function of time for different force fields and counter-ions in Fig. 2. It is seen that the area/lipid levels out and oscillates around a constant value in the time range of 120-180 ns, which implies that the system is equilibrated. We have considered the regular CHARMM27 all-atom force field $^{27}$ with sodium as counter-ion (AANa) and calcium as counter-ion (AACa), and a hybrid force field by Hénin et al. ${ }^{25}$ specifically designed for PG lipids. The latter is a combination of the CHARMM27 all-atom force field and a united atom (UA) force field in which the methylene groups in the aliphatic chains have been replaced by pseudo-atoms. We see that the

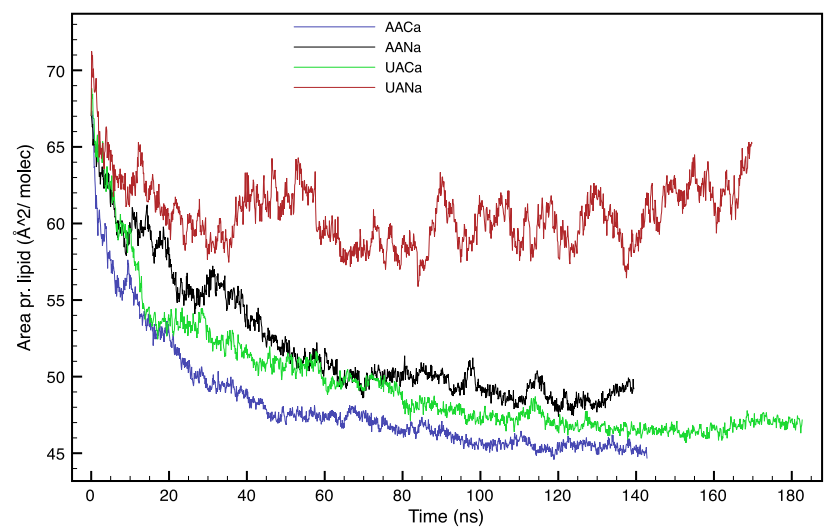

FIG. 2. The area/lipid in a simulation of the DMPG lipid bilayer membrane at $310 \mathrm{~K}$ and 1 atm as function of time with the all-atom CHARMM27 force field and monovalent sodium counter-ions (AANa), all-atom force field with divalent calcium counter-ions (AACa), hybrid model with sodium counter-ions (UANa), and hybrid model with calcium counter-ions (UACa). 
hybrid model (UANa) at equilibrium (120-170 ns) gives an area of $61.3 \pm 1.7 \AA^{2}$ with a monovalent counter-ion and a significantly smaller area of $46.7 \pm 1.0 \AA^{2}$ with a divalent counter-ion, which are within the ranges reported by Marra. ${ }^{28}$ The all-atom model (AANa) with a sodium counter-ion gives too small an area/lipid, whereas the area/lipid with the calcium counter-ion (AACa) is roughly the same as with the hybrid force model. This result illustrates the great importance of the divalent counter-ion in determining the area/lipid.

We also did a simulation with the sodium counter-ion and a $0.1 \mathrm{M} \mathrm{NaCl}$ solution $(\mathrm{UANaCl})$ to evaluate the effect of the salt on the area/lipid, which is shown as a function of time in Fig. 3. There does not seem to be a significant difference between the UANaCl and UANa results. Averaged over the last $20 \mathrm{~ns}$ of the simulations, we find an area of $59.2 \pm 1.0 \AA^{2}$ for $\mathrm{UANaCl}$ and $61.3 \pm 1.7$ for UANa.

In our simulations of the DMPC lipid bilayer membrane, we also found that the CHARMM27 force field gave a too small area/lipid. Therefore, we used a slightly modified version of the CHARMM27 force field where some charges in the head group were altered to improve agreement with experimental values of the area/lipid. ${ }^{29} \mathrm{We}$ assumed that the modifications introduced for the 1,2-dipalmitoyl-sn-glycero-3phosphocholine (DPPC) lipid bilayer carry over to the DMPC bilayer, which they seem to do as far as we can determine. After having completed our DMPC work and begun those on DMPG, we became aware of a new force field, which, for several PCs, ${ }^{30}$ gives an excellent agreement between simulated and experimental area/lipid. However, this new force field has not been tested on PGs; so, we decided to continue the DMPG simulations with the hybrid model, which has shown to give reasonable agreement with experimental values of the area/lipid with both monovalent and divalent counter-ions.

\section{EFFECTS OF THE COUNTER-ION}

The dramatic drop in the area/lipid with a divalent counter-ion together with the smaller fluctuations in the area seems to indicate that the state of the system changes from the fluid to the gel phase, when a divalent counter-ion replaces a monovalent one. This hypothesis was tested by a calculation of the NMR-order parameter, ${ }^{31}$ which is a measure of the order in the aliphatic chains. It is higher in the gel phase than in the fluid phase. Because the hybrid force field uses pseudo-atoms along the chains, we need to "reconstruct" the methylene hydrogen atom positions. Following the procedure given by Egberts et al., ${ }^{32}$ for determining the H-positions, we calculate an NMR order parameter, which is analogous to that for an all-atom model. The results in Fig. 4 clearly show a higher order in the UACa system characteristic of what is found in gel phases and a lower order in the UANa system characteristic of what is found in a fluid phase.

Further evidence of a phase change is that the translational diffusion of the lipids is slow as evidenced by the width of the quasielastic scattering discussed below.

There do not exist x-ray diffraction data on the DMPG system, but Sun et al. ${ }^{33}$ have conducted $\mathrm{x}$-ray diffraction measurements on the gel phase of a DPPC lipid bilayer membrane. They find that the intensity of diffuse scattering is at least as great as that of Bragg scattering, implying that there is still considerable disorder in the gel phase even though the chains are ordered. As an illustration of such structures, we have shown snapshots from the simulations of the fluid and gel phases in Fig. 5. As in the x-ray experiments, we find both ordered and disordered regions in the gel phase as opposed to the disordered fluid phase.

No attempt has been made to calculate the structure factor of the system in the gel phase because our system is too small to give meaningful results, especially in the presence of both ordered and disordered regions, and because there are no experimental data with which to compare.

So our assignment of the UACa system as being a gel phase is based primarily on the NMR order parameter, following the practice in the literature, the reduction in the area per lipid, and the slower diffusion of the lipids.

This transition from the fluid to the gel phase at $310 \mathrm{~K}$ means that the gel-to-fluid transition temperature increases upon addition of a divalent counter-ion. This conclusion agrees with experiments, which report ${ }^{34}$ that the gel-to-fluid transition temperature increases about $20{ }^{\circ} \mathrm{C}$ from $\sim 24^{\circ}$ to $\sim 44^{\circ}$ with the replacement of a monovalent counter-ion by a divalent one. Also shown in Fig. 4 is the order parameter in the presence of a $0.1 \mathrm{M} \mathrm{NaCl}$ solution. It is slightly higher than without salt, but the membrane is still in the fluid phase. Further experimental evidence for the effect of the divalent cation stabilizing the gel phase is provided by Garidel et al. ${ }^{35}$ who use infrared spectroscopy to infer chain ordering in the DMPG membrane.

In order to understand the driving force behind the phase transition, we have determined the pair distribution function

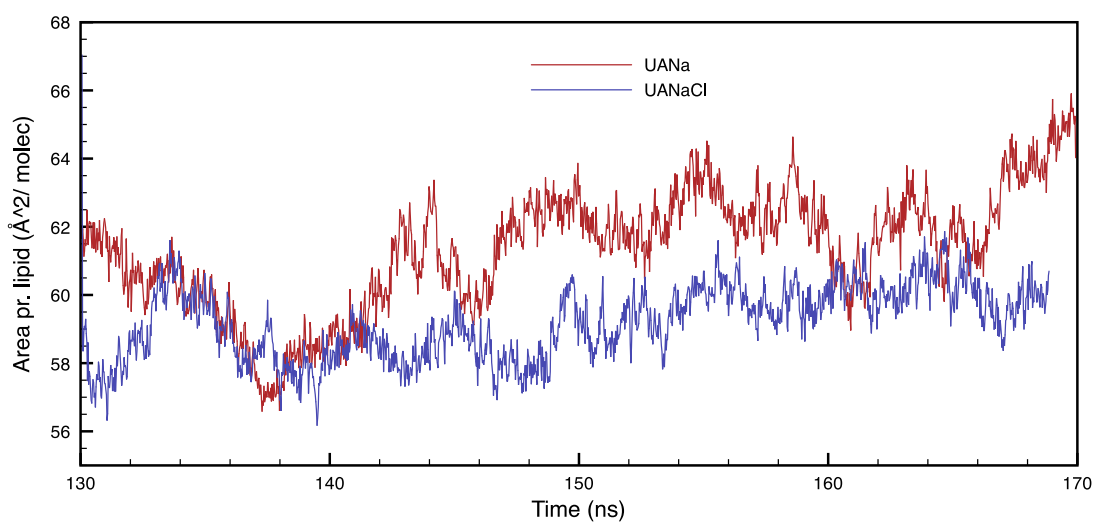

FIG. 3. The area/lipid in the simulation of the DMPG lipid bilayer at $310 \mathrm{~K}$ and $1 \mathrm{~atm}$ with monovalent sodium as the counter-ions in a solution without salt (UANa) and in a $0.1 \mathrm{M} \mathrm{NaCl}$ salt solution $(\mathrm{UANaCl})$. 


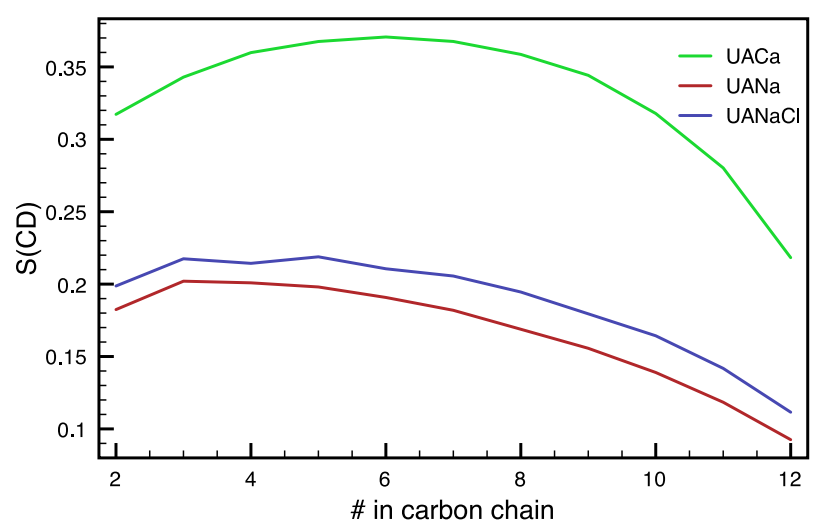

FIG. 4. The NMR-order parameter for the aliphatic chains in DMPG at $310 \mathrm{~K}$ and $1 \mathrm{~atm}$ as a function of carbon atom in the chains. Results for the membrane with divalent counter-ions (UACa), monovalent counter-ions (UANa), and monovalent counter-ions and a $0.1 \mathrm{M} \mathrm{NaCl}$ solution are shown.

for the counter-ions around the phosphate atoms in the lipids. They show the first shell peaked at $\sim 3.5-3.6 \AA$ from the phosphorous atom with a width such that all first shell atoms are found at distances less than $4 \AA$. We then determined the fraction of counter-ions that are within the $4 \AA$ distance from the phosphorous atom in only one lipid and from the phosphorous atoms in two different lipid molecules at the same

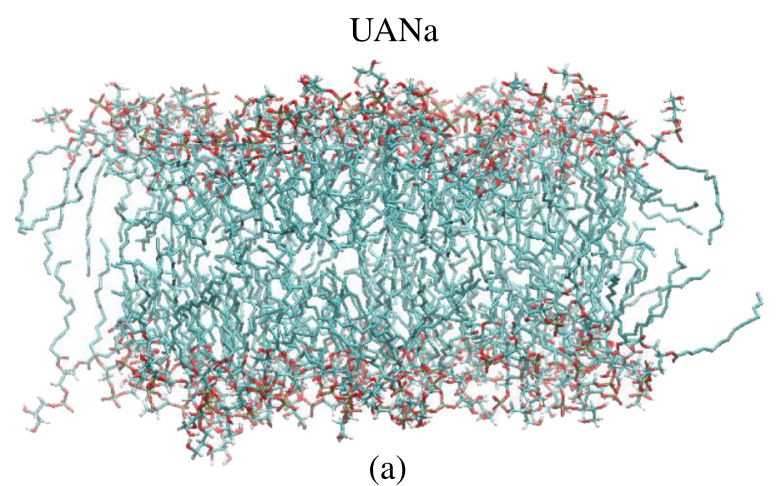

(a)

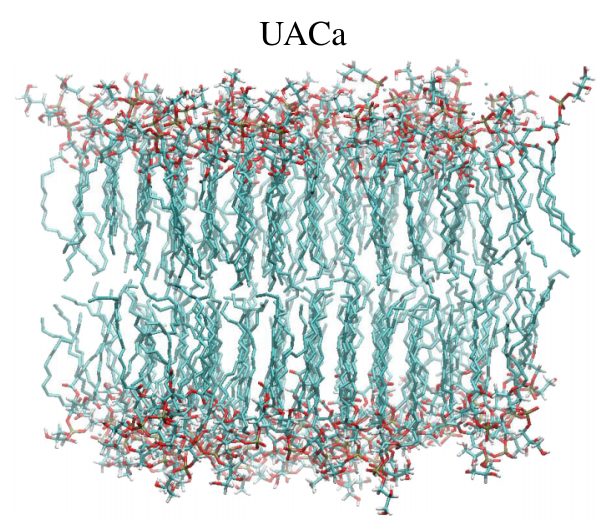

(b)

FIG. 5. Snapshots showing side views in a direction perpendicular to the surface normal $z$ of the fluid and gel phases: (a) UANa system in the fluid phase, (b) UACa system in the gel phase. Water molecules have been removed to facilitate viewing of the partial lateral order of the aliphatic chains of the lipid molecules. The picture of the fluid phase shows a disordered phase independent of the azimuth, whereas the partially ordered regions in the gel phase appear periodically in the azimuth alternating with azimuthal ranges where the structure appears to be disordered. time. For the UANa system, $14 \%$ of the sodium ions were on average in close contact with two lipids simultaneously compared to $91 \%$ of the calcium ions. This significant difference shows the higher affinity of the calcium ion to the lipids and the capability of the divalent counter-ion to link neighboring lipids via a calcium bridge and thereby create more order. This mechanism appears to provide the driving force for the phase transition. The result is consistent with NMR results ${ }^{36}$ showing that sodium only binds to one lipid and calcium binds to two lipids and thereby creates more order.

The temperature-driven fluid-to-gel transition in the zwitterionic DMPC bilayer membrane is only accompanied by $\mathrm{a} \sim 8 \%{ }^{37}$ reduction in the area/lipid as opposed to the calciumion-driven $\sim 25 \%$ effect in the DMPG membrane, when a monovalent counter-ion is exchanged by a divalent one. To our knowledge, the reduction of the area/lipid in a temperaturedriven transition for the DMPG bilayer membrane with a monovalent counter-ion has not been determined but is probably similar in magnitude to the effect in the DMPC membrane.

\section{ATOMIC DENSITY PROFILES IN THE DIRECTION OF THE MEMBRANE SURFACE NORMAL}

The density profile in the direction of the surface normal (z-axis) has been determined for all water molecules, counterions, and for the following lipid-molecule atoms (see also Fig. 1(a) for identification): the phosphorous atom, a carbonyl carbon atom (C Ester) that links one of the aliphatic chains to the glycerol backbone, the middle-carbon atom ( $\mathrm{C} \mathrm{Mid)} \mathrm{in}$ one of the aliphatic chains, and the methyl group carbon atom at the end of one of the aliphatic chains (C Tail). The space along the $z$-axis was divided into $0.2 \AA$ wide slabs with the center of the bilayer located at $z=0$. The $z$-coordinates of the center-of-mass of the water molecules and of the selected atoms, respectively, are used to assign them to the bins. Data were obtained for 8000 time frames in a 4-ns time-block and converted to a number density as function of $z$. The number density profiles are presented in Fig. 6 and, because the number density of water molecules is much larger than that of the selected atoms and counter-ions, we have shown a blow-up of the profiles in Fig. 7 to clarify details of the membrane interior.

Qualitatively, the density profiles are very similar to those found for the DMPC system ${ }^{17}$ with rather broad distribution functions and a full-width-at-half-maximum (FWHM) equal to 6-8 $\AA$. We note that the water profile is independent of $z$ outside the membrane in the $z$-range $\pm(25-40) \AA$. These regions therefore appear to have bulk-like water with a mass density of $1.02 \mathrm{~g} \mathrm{~cm}^{-3}$ equivalent to the given number density. It agrees with the mass density that we find for bulk TIP3P water at $310 \mathrm{~K}$ and $1 \mathrm{~atm}$ and is in fair agreement with the experimental value of $0.993 \mathrm{~g} \mathrm{~cm}^{-3} \cdot 38$

There are some obvious differences between the density profiles, which may be related to the system with the calcium counter-ion being in the gel phase and that with the sodium counter-ion being in the fluid phase. First, the profiles are somewhat narrower for the system with the calcium counterion. Second, note that with the calcium counter-ion present, 


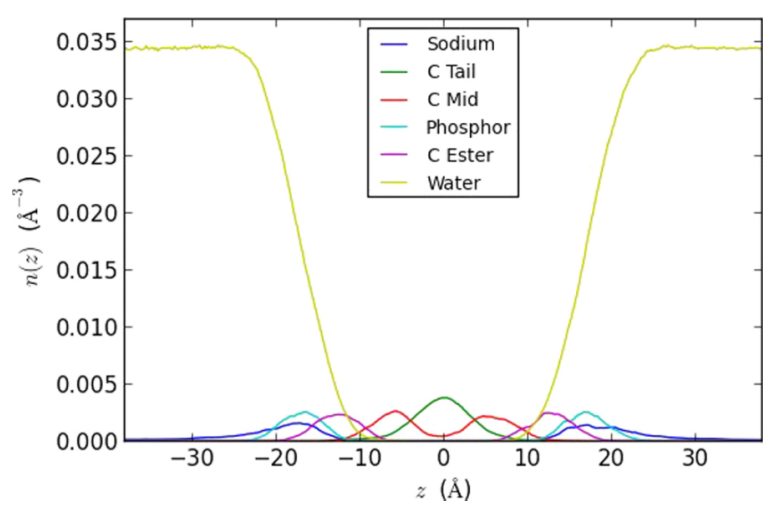

(a)

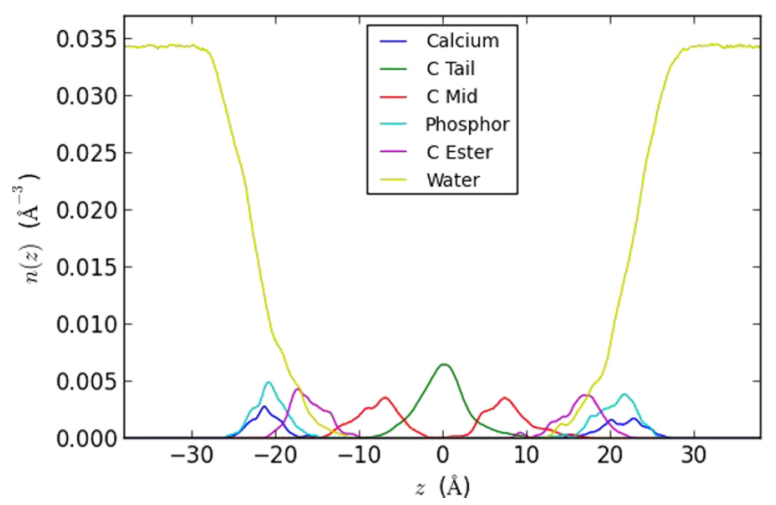

(b)

FIG. 6. The density profiles of water, counter-ions, and selected DMPG lipid atoms (as identified in Fig. 1(a)) in the direction of the surface normal to the membrane ( $z$-axis) at $310 \mathrm{~K}$ and $1 \mathrm{~atm}$ : (a) with sodium counter-ions and (b) with calcium counter-ions. Note that the full extent of the bulk water region is not shown. For the system with sodium counter-ions, it extends to $z \sim 49 \AA$ and with calcium counter-ions to $z \sim 58 \AA$.

the profile for the $\mathrm{C}$ mid atom (red curve) is at zero at the center of the membrane $(\mathrm{z}=0)$ but non-zero with the sodium counter-ion, indicating more chain order in the system with the calcium counter-ion. The water profiles show that there is more water in the fluid-phase membrane (UANa) than in the gel-phase membrane (UACa), which is consistent with the water layer above the gel phase membrane being about $5 \AA$ thicker than above the fluid phase membrane as mentioned in Section II A. Water has been squeezed out of the membrane region into the bulk water phase. There is an interesting difference in the distribution of the counter-ions. In the UACa system, the calcium ion distribution (blue curve) overlaps closely with the phosphorous atom distribution (light blue curve), whereas the sodium ion distribution (blue curve) in the UANa system has a "tail" extending out in the bulk water region. This behavior is consistent with our finding that most calcium ions are bound to two phosphate atoms in neighboring lipids, while most sodium ions are only bound to one lipid. The difference in the distribution of the counterions is of importance for the electrostatic potential in the membrane system and its extension outside the membrane as will be discussed below. The distribution function for the tail carbon atom (green curve) is centered at the membrane mid-plane as expected and for the carbonyl carbon (C ester, magenta curve) between the phosphorous and middle-carbon distribution functions.

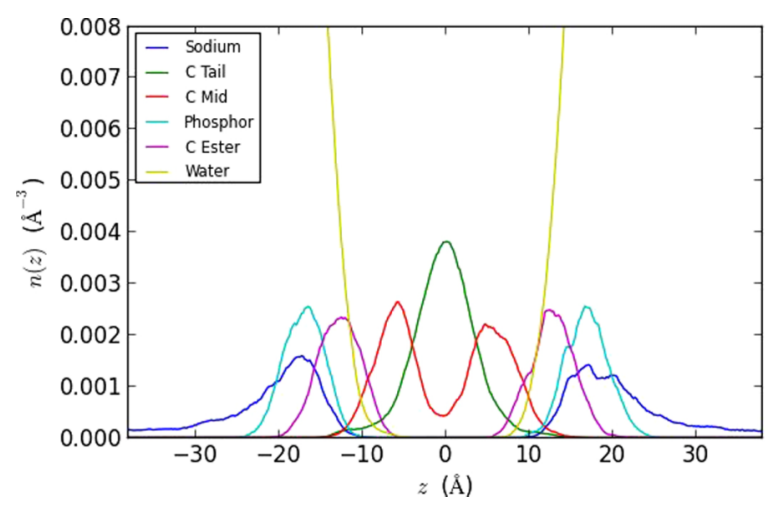

(a)

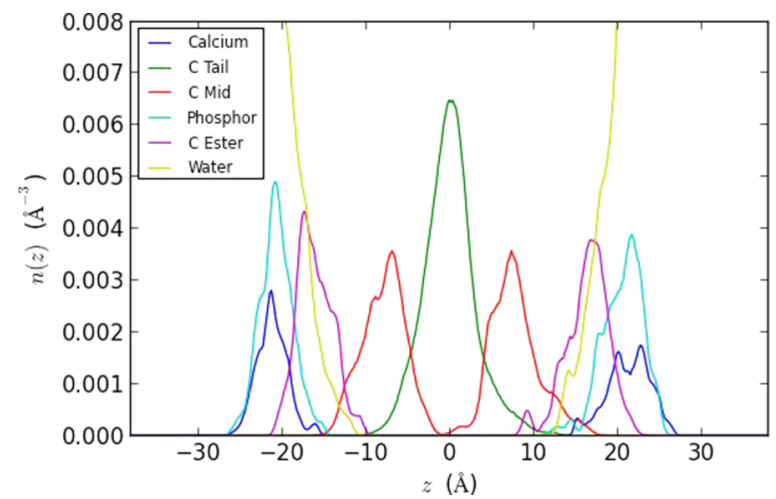

(b)

FIG. 7. A blow-up of the profiles in Fig. 6 to make details in the membrane region clearer: (a) with sodium counter-ions and (b) with calcium counterions.

No atoms from the glycerol unit in the head group have been included. Because the orientation of the head group, as mentioned in Section I, is almost parallel to the membrane surface, there will be a great overlap between the distribution functions of the atoms in the glycerol subunit in the head group and that of the phosphate atom; so, the extension of lipid atoms into the bulk-water region will only be slightly larger than shown in Fig. 7.

We have determined the number of water molecules per lipid required for full hydration of the DMPG membrane from the volume of the hydrated membrane. The membrane is fully hydrated because there is an excess of bulk water molecules above the membrane. In experiments, the size of the membrane is determined by $\mathrm{x}$-ray measurements, and the number of water molecules is calculated from different assumptions about the volume of lipid and water molecules. Here we use the water profile to determine the dimension of the membrane in the $z$-direction perpendicular to the membrane surface. The extent of the membrane in the $z$-direction is defined as the point where the water profile becomes independent of $z$. For both leaflets, this point is seen to be roughly halfway into slab 3 in Fig. 8. The lateral extent of the membrane is given by the simulation box size in the $x$ - and $y$-directions. Then the amount of hydration water is calculated as the number of water molecules in those slabs or parts thereof within the membrane so defined, and we find 9.7 water molecules per lipid in the gel phase and 17.5 molecules in the fluid phase.

As a check of the procedure, we made a similar determination of the number of water molecules in a fully 


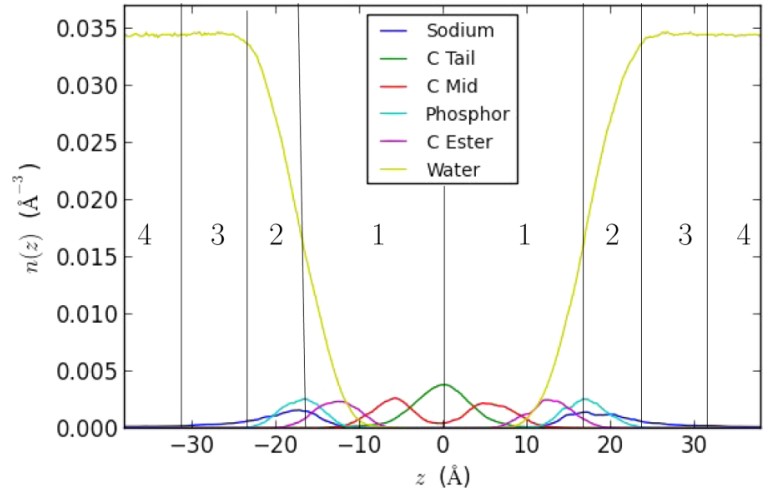

(a)

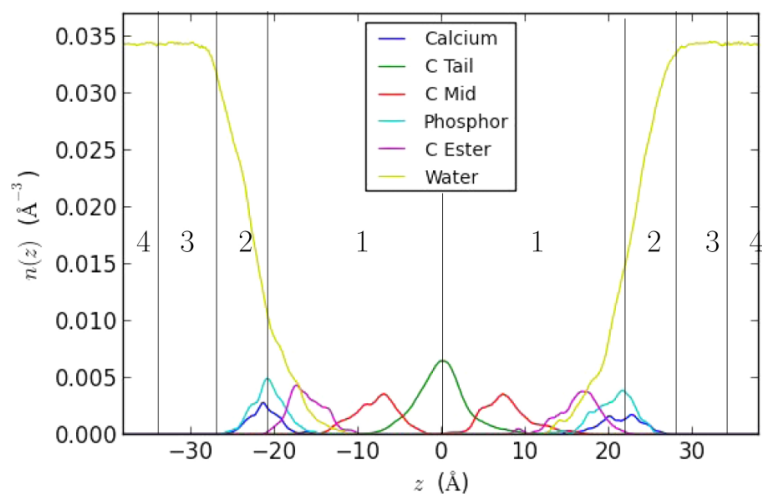

(b)

FIG. 8. Definition of the slabs used in the calculation of the diffusion constant for water as a function of its depth in the membrane: (a) with sodium counter-ions and (b) with calcium counter-ions.

hydrated DMPC membrane using our results in Ref. 17 and found $\sim 28$ water molecules per lipid in good agreement with the reported 27 water molecules. ${ }^{20}$

\section{DIFFUSION CONSTANTS}

\section{A. Mean-square-displacement}

The diffusion constants of the lipid and the water molecules in various regions of the membrane have been calculated from mean-square displacements following the procedure described in our DMPC work. ${ }^{17}$ The division of the membrane in regions or slabs is shown in Fig. 8 for the systems with the two different counter-ions. We have used the position of the phosphorous atom to define the inner region slab 1 from the center of the membrane at $z=0$ to the phosphorous peak position. Beyond it, there are three $7.5-\AA$ thick slabs as we move out into the outer membrane and bulk-water regions. The mean-square displacement calculations were done with an 80-ps time window and averaged over 30 choices of time origins. From the slope in a plot of the mean-square-displacement as a function of time, we obtain the diffusion constants, as described in Ref. 17. The results are compiled in Table I. For reference, the diffusion constant of bulk TIP3P water at $310 \mathrm{~K}$ and 1 atm was determined to be $3.0 \cdot 10^{-5} \mathrm{~cm}^{2} \mathrm{~s}^{-1}$ in a separate simulation of bulk water.
TABLE I. Diffusion constants for water at $310 \mathrm{~K}$ and $1 \mathrm{~atm}$ as a function of its position in the DMPG membrane as defined by the slabs in Fig. 8. For reference, our simulations give the diffusion constant of bulk TIP3P water to be $3.0 \cdot 10^{-5} \mathrm{~cm}^{2} \mathrm{~s}^{-1}$, and the center-of-mass diffusion constants for the lipids to be $1.0 \cdot 10^{-7} \mathrm{~cm}^{2} \mathrm{~s}^{-1}$ and $5.0 \cdot 10^{-8} \mathrm{~cm}^{2} \mathrm{~s}^{-1}$ for the fluid phase membrane (UANa) and the gel phase membrane (UACa), respectively.

\begin{tabular}{|c|c|c|c|c|}
\hline & Slab 1 & Slab 2 & Slab 3 & Slab 4 \\
\hline System & $10^{-5} \mathrm{~cm}^{2} \mathrm{~s}^{-1}$ & $10^{-5} \mathrm{~cm}^{2} \mathrm{~s}^{-1}$ & $10^{-5} \mathrm{~cm}^{2} \mathrm{~s}^{-1}$ & $10^{-5} \mathrm{~cm}^{2} \mathrm{~s}^{-1}$ \\
\hline UANa & $0.23 \pm 0.003$ & $1.06 \pm 0.01$ & $2.83 \pm 0.02$ & $3.00 \pm 0.02$ \\
\hline UACa & $0.063 \pm 0.0005$ & $0.54 \pm 0.005$ & $2.95 \pm 0.03$ & $2.99 \pm 0.02$ \\
\hline $\mathrm{UANaCl}$ & $0.20 \pm 0.003$ & $1.00 \pm 0.01$ & $2.60 \pm 0.03$ & $3.00 \pm 0.02$ \\
\hline
\end{tabular}

We see that the diffusion constant in slab 4 is practically identical to that in bulk water, so the influence of the lipid bilayer membrane on the mobility of water is negligible in that region. In slab 3, the diffusion constant of water is slightly reduced from that in bulk water similar to what we saw in the corresponding layer 1 in our DMPC work. ${ }^{17}$ In slabs 1 and 2 , a strong influence of the membrane on the mobility of water is evident. The effect of the phase transition from the fluid phase (UANa) to the gel phase (UACa) is quite strong, reducing the diffusion constant of water by a factor 2 relative to the fluid phase. The reduction of the diffusion constant in slab 1 is about a factor of 13 relative to the bulk diffusion constant in slab 4 for the fluid system (UANa) and a factor of about 50 for the gel system (UACa). The diffusion constants in the outer slab 2 are up by about a factor 5-9 relative to slab 1 , and the diffusion constants in slab 3 are up by a factor of 3-5 relative to slab 2 .

For the fluid DMPC system, ${ }^{17}$ we also found a reduction in the diffusion constant of water in the inner membrane by about a factor of 10 relative to the bulk diffusion constant. The comparison with the DMPC results depends to some extent on the way we define the slabs, so a comparison should be limited only to the gross features.

We have also determined the diffusion constants for the center-of-mass motion of the lipids and found them to be $1.0 \cdot 10^{-7} \mathrm{~cm}^{2} \mathrm{~s}^{-1}$ for DMPG in the fluid phase (UANa) at $310 \mathrm{~K}$ and $1 \mathrm{~atm}$ and $5.0 \cdot 10^{-8} \mathrm{~cm}^{2} \mathrm{~s}^{-1}$ for DMPG in the gel phase (UACa). For DMPC in the fluid phase at $303 \mathrm{~K}$, we found the diffusion constant to be $9.76 \cdot 10^{-8} \mathrm{~cm}^{2} \mathrm{~s}^{-1}$ in good agreement with the result for DMPG in the fluid phase, considering that the temperature of DMPG is slightly higher at $310 \mathrm{~K}$. The smaller diffusion constant of DMPG with the calcium counter-ion is another manifestation of the system being in the gel phase.

\section{B. Quasielastic incoherent scattering function}

We have calculated the quasielastic incoherent neutron scattering function for water in the DMPG membrane system to make comparison with quasielastic neutron scattering experiments on single-supported DMPG bilayer membranes. Following the procedure given in our DMPC paper, ${ }^{17}$ we have used the spherically averaged intermediate scattering function for the water molecules that are free to translate and rotate in all three Cartesian directions. The time window used in 
the calculations is $2 \mathrm{~ns}$ corresponding to an energy resolution $\sim 1 \mu \mathrm{eV}$ as in the experiments, and the data were recorded with a time interval of $0.5 \mathrm{ps}$ that allowed a dynamical range of $\sim 4.1 \mathrm{meV}$, well above that of the spectrometer used in the experiments $(\sim 120 \mu \mathrm{eV})$. The incoherent scattering functions were calculated for a range of wave vectors $0.2 \leq q \leq 1.3 \AA^{-1}$ because the $q$-dependence of the half-width-at-half-maximum (HWHM) of the scattering function indicates what kind of diffusive motion is involved. For random translational diffusion motion, for example, the relation between the HWHM and $q^{2}$ is linear with the slope being equal to the translational diffusion constant, whereas it is independent of $q$ for pure rotational diffusive motion. If both kinds of motion occur on the same time scale, the dependence on $q$ is more complex.

As discussed in Refs. 39 and 40, there will be an elastic contribution to the scattering function $S(q, \omega)$, if the calculated intermediate scattering function $F(q, t)$ is non-zero at $t=t_{\text {window }}$, the time window used in the calculation of $F(q, t)$. When we calculate $S(q, \omega)$ by a time-Fourier transform of $F(q, t)$, we have found numerical noise in the data at small energies, if there is an elastic contribution. We therefore subtract $F\left(q, t_{\text {window }}\right)$ from $F(q, t)$ before doing the timeFourier transformation of the modified intermediate scattering function, which eliminates the problem with the numerical noise.

When experimental quasielastic scattering data are analyzed, they are fit to a sum of two Lorentzians (representing scattering from random translational diffusion), a delta function in the energy (the elastic contribution), and a background. In the simulations, we fit the calculated scattering function to only a sum of Lorentzians because an elastic contribution has been eliminated, and there is no background scattering.

At all $q$, we were able to fit the calculated scattering functions to two Lorentzians, a broad one representing "fast" water molecules and a narrow one representing more "slowly" moving water molecules. As the "fast" and "slow" terms are somewhat imprecise, each Lorentzian component represents an average over water molecules whose motion spans a range of time scales. The ranges of the two components do not overlap.

In Fig. 9(a), we have plotted the HWHM ( $\left.\Gamma_{\text {broad }}\right)$ of the broad (fast) component in the Lorentzian fit for UANa and UACa simulations as function of $q^{2}$. The linear relationship shows that the broad (fast) component is associated with random diffusive translational motion, and, from the slope, we find the diffusion constants to be $2.36 \cdot 10^{-5} \mathrm{~cm}^{2} \mathrm{~s}^{-1}$ in the UANa system and $2.53 \cdot 10^{-5} \mathrm{~cm}^{2} \mathrm{~s}^{-1}$ in the UACa system. At first sight, it may be surprising that we find a larger diffusion constant for the UACa system, which is in the gel phase where the mean-square displacement calculations of the water molecules gave a smaller diffusion constant (Table I). However, to compare the simulation results with the observed quasielastic neutron spectra, we are calculating the diffusion constant averaged over all the water molecules in the simulation cell. Because there is more bulk water in the UACa simulation (some water has been squeezed out of the membrane in its gel phase), the

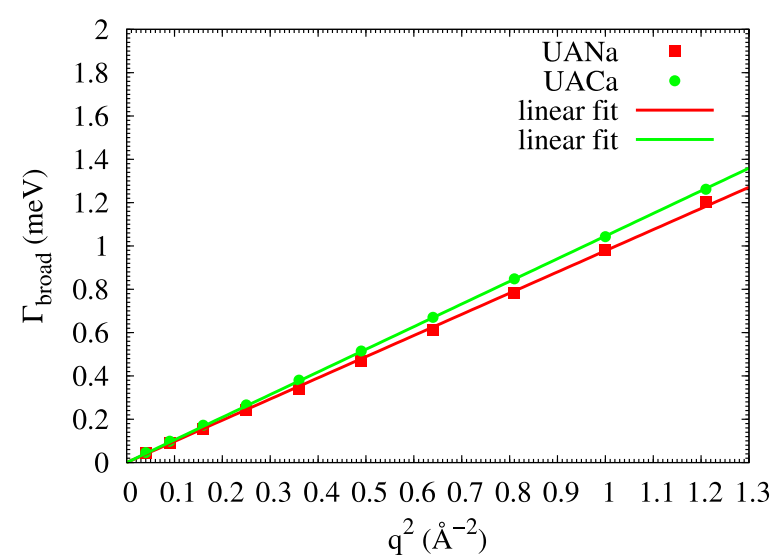

(a)

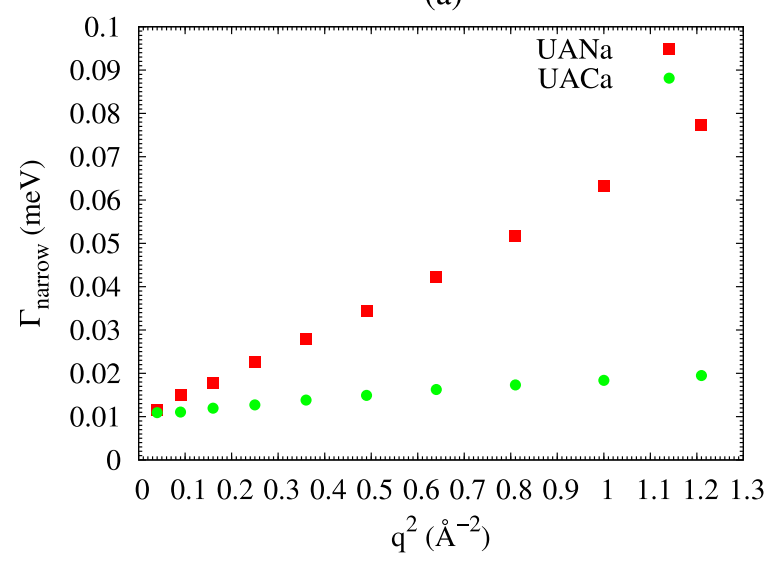

(b)

FIG. 9. HWHM of the broad component $\left(\Gamma_{\text {broad }}\right)$ in the Lorentzian fit to the quasielastic scattering function at $310 \mathrm{~K}$ and $1 \mathrm{~atm}$ as a function of the square of the wave vector transfer $q^{2} \AA^{-2}$ for (a) DMPG bilayer with sodium as counter-ion (UANa) and (b) DMPG bilayer with calcium as counter-ion (UACa).

diffusion constant averaged over the whole sample increases. The diffusion constant determined from the broad Lorentzian in a scattering experiment is always a weighted average over the different "fast" diffusion time scales in the system. It is smaller than the value that we have obtained in simulations of bulk water $\left(\sim 3.0 \cdot 10^{-5} \mathrm{~cm}^{2} \mathrm{~s}^{-1}\right)$, which indicates that the broad Lorentzian intensity is not completely dominated by bulk water. If we increase the amount of water, the diffusion constant will approach that of bulk water; and, from the width of the broad Lorentzian, we will not be able to detect the presence of water molecules with a lower mobility.

The HWHM ( $\left.\Gamma_{\text {narrow }}\right)$ of the narrow Lorentzian (slow) component in the scattering function is shown in Fig. 9(b) and represents slow-moving water molecules interacting with the lipid molecules. The departure from a simple linear relationship of $q^{2}$, like that found for the broad component $\Gamma_{\text {broad }}$, indicates that the motion associated with $\Gamma_{\text {narrow }}$ is more complex and not just a simple translational diffusion. The almost $q$-independent $\Gamma_{\text {narrow }}$ for the UACa system could be explained by molecules performing rotational (torsional) motions. If we assume that the slow-moving water molecules follow the motion of the lipids because they are "bound" to them, then their motion will be a superposition of the 
translational motion of the center-of-mass of the lipids and the torsional (wagging) motions of the various groups in the lipid with respect to the center-of-mass as discussed in our DMPC paper. ${ }^{17}$ In this case, the $q$ dependence of $\Gamma_{\text {narrow }}$ in the UANa system is larger because the center-of-mass diffusion constant of the lipids is larger than in the UACa system, resulting in a stronger weight of translational motion in the scattering function and hence a larger $q$ dependence.

We have not made a calculation of the scattering function for the lipid molecules, but we may make a rough estimate of $\Gamma$ for the translational center-of-mass motion based on the mean-square displacement calculations of the center-of-mass diffusion constant. For $q=1$, we get $\Gamma=4.15 \mu \mathrm{eV}$ for the UANa system and $2.07 \mu \mathrm{eV}$ for the UACa system. For both systems, these values are smaller than for the types of water in Fig. 9(b) which follow the motion of the lipid head-groups. In addition to translational motion of the lipids they also have rotational and torsional motions resulting in the larger $\Gamma$ values for the water molecules.

In the fit of the calculated scattering functions to the two Lorentzians, there is a width and an intensity parameter associated with each one. The Lorentzian intensity measures the number of water molecules moving on the slow (narrow component) and fast (broad component) time scales. Therefore, it may be used to determine the fraction of the water molecules that move on, for example, the slow time scale. However, this fraction depends on the total number of water molecules in the system and will become smaller, the more bulk molecules (moving on the fast time scale) there are in the system. So, in order to obtain a more "universal," sizeindependent, measure that allows us to compare simulation and experimental results, we may convert the fraction to the number of water molecules per lipid molecule that move on a slow time scale. With 6760 water molecules and 128 lipid molecules, it is easy to determine that number.

In the determination of the fraction of water molecules that move on the slow time scale, it is important to remember that there also may be elastic scattering from some water molecules. Because it is difficult to estimate the intensity of their elastic scattering, we have proceeded as follows. Generally, the elastic scattering is appreciable only at "small" $q$; so, we base our calculation of the fraction of slow-moving molecules on the narrow and broad Lorentzian intensities at "large" $q$. To quantify the meaning of "small" and "large" $q$, we monitor the fraction of slow-moving water molecules as a function of $q$ and, when the results become independent of $q$, we assume that the elastic contribution to the scattering has dropped to zero. For the UACa system, we find 2.1 slowmoving water molecules per lipid at $q \geq 0.75 \AA^{-1}$ and 4.2 water molecules/lipid in the UANa system at $q \geq 0.75 \AA^{-1}$. As discussed above, the fewer water molecules per lipid in the UACa system are probably related to the squeezing out of water molecules from the membrane, which is in the gel phase.

\section{COMPARISON WITH EXPERIMENTS}

A comprehensive report of quasielastic neutron measurements investigating the dynamics of water in proximity to single-supported DMPG membranes will be published separately. ${ }^{12}$ Here we describe some preliminary results for comparison with those obtained above from the MD simulations. As in the case of previous quasielastic neutron scattering (QENS) investigations of DMPC membranes, ${ }^{11,18}$ single-bilayer DMPG membranes were deposited onto both sides of single-crystal silicon wafers. A stack of 100 such wafers comprised a sample. Quasielastic spectra have been obtained on two different backscattering spectrometers: the high flux backscattering spectrometer (HFBS) at The NIST Center for Neutron Research ${ }^{41}$ and backscattering silicon spectrometer (BASIS) at the Spallation Neutron Source, Oak Ridge National Laboratory. ${ }^{42}$

The QENS measurements have been conducted on two samples both of which had a higher level of hydration than in the simulation. As described in Ref. 18, we express the amount of water in a sample by the thickness of an equivalent slab of water having the bulk density located on each side of a wafer. The thickness was $\sim 71 \mathrm{~nm}$ for a so-called "wet" sample and $\sim 23 \mathrm{~nm}$ for a "dry" sample. The QENS measurements were done at room temperature and below because we were primarily interested in investigating the dynamics of different components of the membrane-associated water that could be distinguished by their freezing behavior. ${ }^{11,12,18}$

We fit the QENS spectra by folding the instrumental resolution function with a scattering law composed of three terms: a delta function corresponding to the elastic scattering plus two Lorentzians representing the quasielastic scattering. ${ }^{11,12,18}$ Both Lorentzian components were found to have a HWHM that has a linear dependence on $q^{2}$ for $q \leq 0.9 \AA^{-1}$ whose slope could be used to compute a diffusion coefficient. However, it is difficult to compare quantitatively the diffusion coefficients inferred from the simulation with those from the experiment because the simulations cannot be done easily at the lower temperatures of the experiments due to the very long equilibration times. Another consideration in comparing the diffusion coefficients inferred from the simulation and experiment is the limited dynamic range of the spectrometers used in the measurements. For example, at room temperature, the width of the quasielastic spectra near $q=0.9 \AA^{-1}$ becomes comparable to the dynamic range of the BASIS spectrometer $( \pm 120 \mu \mathrm{eV})$, increasing the uncertainty in the determination of the diffusion coefficient. Finally, because the single-supported membranes investigated experimentally are believed to be in the gel phase, ${ }^{11,18}$ we restrict comparison to the simulation results of the UACa system (the UANa system is in the fluid phase).

At $295 \mathrm{~K}$, analysis of the broad Lorentzian component in the observed QENS spectra of the wet DMPG sample yields a diffusion constant $D=1.7 \cdot 10^{-5} \mathrm{~cm}^{2} \mathrm{~s}^{-1} \cdot{ }^{12}$ Assuming the same temperature dependence of $D$ as found in NMR measurements on bulk water ${ }^{43}$ and extrapolating to the simulation temperature of $310 \mathrm{~K}$, we estimate $D$ $=2.3 \cdot 10^{-5} \mathrm{~cm}^{2} \mathrm{~s}^{-1}$ compared to the value of $2.5 \cdot 10^{-5} \mathrm{~cm}^{2} \mathrm{~s}^{-1}$ inferred from analysis of the broad Lorentzian component in the simulated spectra (see Fig. 9(a)). We believe that the sources of error discussed above as well as the error in extrapolation to higher temperature could account for the difference in $D$ obtained in the experiment and simulation. Because both $D$ values represent an average over all water in 
the system, including water inside the membrane, we expect them to be close to but less than for bulk water. Extrapolating the NMR measurements on bulk water ${ }^{43}$ from $295 \mathrm{~K}$ to the simulation temperature of $310 \mathrm{~K}$ scattering, we obtain $D$ in the range $2.6-2.7 \cdot 10^{-5} \mathrm{~cm}^{2} \mathrm{~s}^{-1}$, which, as expected, is slightly larger than the value inferred from the simulation but smaller than $3.0 \cdot 10^{-5} \mathrm{~cm}^{2} \mathrm{~s}^{-1}$ as found in our simulations of bulk TIP3P water at $310 \mathrm{~K}$ and 1 atm. Because the QENS measurements were done on samples having a higher hydration level than in the simulations, we would expect the value of $D$ inferred from them to be closer to the bulk value. In fact, on cooling the wet DMPG sample to $270 \mathrm{~K}$, where the width of the quasielastic scattering is within the dynamic range of the BASIS spectrometer, we find a $D=0.9 \cdot 10^{-5} \mathrm{~cm}^{2} \mathrm{~s}^{-1}$ in reasonable agreement with values for bulk supercooled water determined by $\mathrm{NMR}^{43}$ and QENS. ${ }^{44}$

A similar analysis of the narrow Lorentzian component in the QENS spectra of both the wet and dry DMPG samples yields a smaller translational diffusion coefficient, which presumably characterizes the dynamics of water closer to the lipid head groups. In this case, the diffusion coefficient has a much weaker temperature dependence than that inferred from the broad Lorentzian component. This behavior is most easily seen for the dry DMPG sample, which has a smaller amount of bulk-like water above the membrane. Extrapolating from the value determined at a temperature of $295 \mathrm{~K}$ for the dry DMPG sample, we estimate a diffusion coefficient of $\sim 4 \cdot 10^{-6} \mathrm{~cm}^{2} \mathrm{~s}^{-1}$ at $310 \mathrm{~K}$, the temperature of the simulation. This value lies within the range of $1.0 \cdot 10^{-6} \mathrm{~cm}^{2} \mathrm{~s}^{-1}$ to $5.0 \cdot 10^{-6} \mathrm{~cm}^{2} \mathrm{~s}^{-1}$ found for the inner slabs 1 and 2, respectively (see Fig. 8), in the simulation of the UACa system (see Table I). Also, for the dry sample at $295 \mathrm{~K}$, we note that the HWHM of the narrow Lorentzian component lies in the range $0-15 \mu \mathrm{eV}$ for $q \leq 0.9 \AA^{-1}$, which overlaps with the range of $10-20 \mu \mathrm{eV}$ found for the narrow Lorentzian in the simulation at $310 \mathrm{~K}$ (see Fig. 9(b)).

Using the same method as in the simulation to estimate the fraction of water molecules undergoing translational diffusion on the slow time scale (see Sec. V), we find that $\sim 18 \pm 8$ water molecules per lipid are contributing to the intensity of the narrow Lorentzian in the dry DMPG sample. The large uncertainty in this value is due to the weak intensity and large width of the broad Lorentzian component, which makes it difficult to distinguish from the background. With this uncertainty, it is difficult to conclude whether the water component undergoing slow translational motion is significantly larger than the 9.1 water molecules per lipid estimated to be required for full hydration of the membrane. However, it is about an order of magnitude larger than the 2.1 water molecules per lipid inferred from the intensity of the narrow Lorentzian component in the simulated quasielastic spectra. It is doubtful that there is sufficient sensitivity to observe a signal originating from such a small component of water in the experiment. We have also seen in Fig. 9(b) that the simulation does not predict a $q^{2}$ dependence of the HWHM at the lowest $q$. Thus, although the water diffusion rate is comparable for the narrow Lorentzian components in the simulated and observed spectra, the two components may not have the same origin.

\section{BINDING OF WATER TO THE PHOSPHOROUS ATOM IN THE HEAD GROUPS}

As in our simulations of the DMPC membrane, we have also analyzed the binding of water to the phosphorous atom in the head groups following the same procedure as described in Ref. 17.

The calculated distribution functions for water molecules as a function of the distance from the phosphorous atoms are shown in Fig. 10 for the DMPG membranes with the (a) sodium and (b) calcium counter-ions, respectively. There are several differences between the distribution functions. In the UACa system, the peak position of the first solvation shell at $\sim 4 \AA$ is shifted to a slightly larger distance and the peak is broader than in the UANa system. Also, the second solvation shell at $\sim 6 \AA$ is quite different in the two systems. These differences reflect the very different environment of the phosphorous atoms with the two different counter-ions as described previously.

When we look at the decay in time of the number of tagged water molecules initially in the first solvation shell around the phosphorous atoms in Fig. 11, there also is a striking difference between the two systems. In the UACa system, some of the water molecules appear to be more strongly bound than in the UANa system as evidenced by the much slower decay rate. This interpretation has been quantified by a fit to the sum of three exponentials, which shows three different average residence times for the tagged water molecules. The first two of these, a short ( $\sim-6 \mathrm{ps})$ and a medium

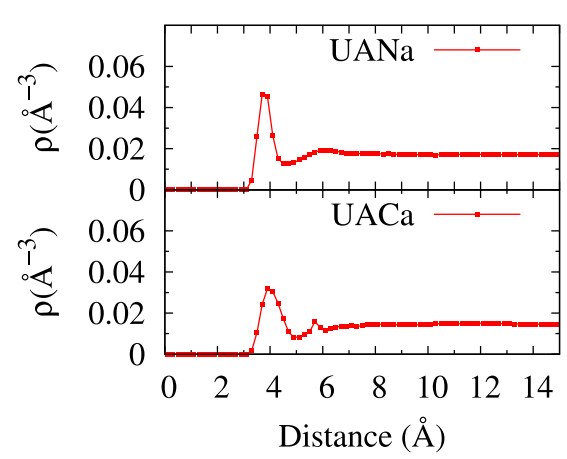

FIG. 10. The water number density $\rho \AA^{-3}$ around the phosphorous atoms in the head groups of the UANa and UACa systems at $310 \mathrm{~K}$ and $1 \mathrm{~atm}$.

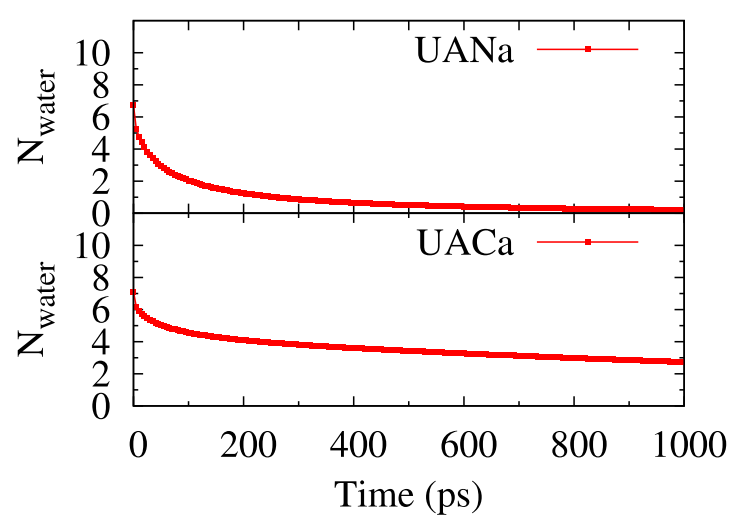

FIG. 11. The decay in time of the number of water molecules, $N_{\text {water }}$, initially in the first solvation shell of the UANa and UACa systems in Fig. 10. 
( $\sim 73-79 \mathrm{ps})$ time are quite similar in the two systems. A third residence time is an order of magnitude larger in the system with a Ca counter-ion ( $2100 \mathrm{ps})$ than in the one with $\mathrm{Na}$ $(\sim 470 \mathrm{ps})$, implying a particularly strong binding in the UACa system.

\section{ORIENTATION OF WATER MOLECULES}

We have already analyzed the influence of the lipid molecules on the mobility of the water molecules. Here we analyze the orientation of the water molecule dipoles. The number of molecules $d N(\theta)$ for which the polar angle $\theta$ of the dipole moment is in the range $(\theta, \theta+d \theta)$ may be written as

$$
d N(\theta)=N f(\theta) \sin \theta d \theta,
$$

where $N$ is the number of molecules and $f(\theta)$ the probability density distribution function in the polar angle $\theta$. Likewise, the number of molecules $d N(\phi)$ for which the azimuthal angle of the dipole moment vector is in the range $(\phi, \phi+d \phi)$ may be written as

$$
d N(\phi)=N g(\phi) d \phi,
$$

where $g(\phi)$ is the probability density distribution function in the azimuthal angle $\phi$. The density distributions are determined by counting the number of molecules $d N(\theta)$ and $d N(\phi)$ for which the polar angle is in the range $(\theta, \theta+\Delta \theta)$ and the azimuthal angle in the range $(\phi, \phi+\Delta \phi)$ with $\Delta \theta=\Delta \phi=1^{\circ}$. The results are averaged over 8000 time frames equivalent to $4 \mathrm{~ns}$. Eqs. (1) and (2) are solved for $f(\theta)$ and $g(\phi)$, respectively.

The results are displayed in Fig. 12 for the UANa system and in Fig. 13 for the UACa system. Note that only slab 1 in these plots is the same as in Fig. 8 for the diffusion coefficient calculations. We have divided the region after slab 1 into 5 slabs, each with a smaller width of $5 \AA$ for a better resolution in $z$ in order to focus on the extent of the electric field into the bulk-like water adjacent to the membrane and its effect on the orientation of the water dipoles. In both systems, we note that the density distribution $\mathrm{g}(\phi)$ is independent of $\phi$ so that no electric field in the azimuthal direction can orient the dipoles. This behavior is expected from the axial symmetry with respect to the surface normal and therefore confirms the consistency of the calculations. The density distributions $f(\theta)$ are quite different for the two systems. One difference is that in the UACa system (Fig. 13), the water dipoles in slab 4 are already randomly oriented in contrast to the UANa system (Fig. 12) where there is still some ordering in slab 6. That is, the electric field in the UANa system extends further outside the membrane than in the UACa system because of the more "diffuse" distribution of the sodium ions around the phosphorous atom (Fig. 7(a)), which reduces the screening outside the double layer formed by the counter-ion and the phosphate group. The "sharper" distribution of the calcium ions (Fig. 7(b)) enhances the screening. In the UANa system, there is a preferred orientation of the dipole moments toward the center of the membrane; a tendency that is reduced as we move further out of the membrane. In the UACa system, there also is a tendency of the dipoles to point inward toward the

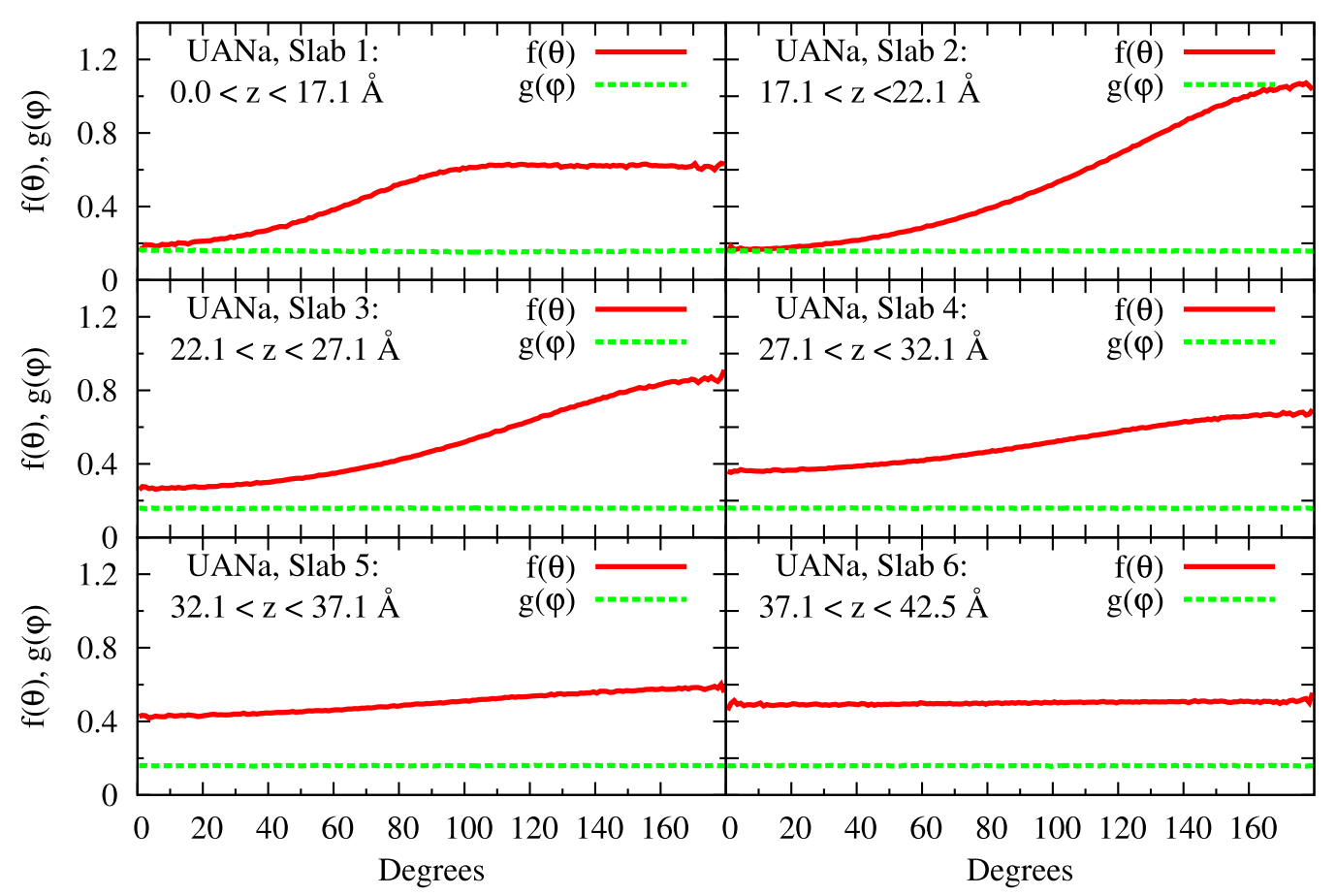

FIG. 12. The polar angle probability density distribution function $f(\theta)$ and the azimuthal angle probability density distribution function $g(\phi)$ in six slabs through the DMPG membrane with sodium counter-ions (UANa) at $310 \mathrm{~K}$ and $1 \mathrm{~atm}$. The results are an average of the distributions in equivalent slabs in the two leaflets. For $\theta=0^{\circ}$, the dipole moment points outwards from the center of the membrane toward the surface; and, for $\theta=180^{\circ}$, the dipole points inward toward the center of the membrane. Note that only slab 1 is the same as in Fig. 8, while the five other slabs are thinner ( $5 \AA$ ) for a better resolution in $z$ and determination of the extent of the region outside the membrane where there still is an orientational effect on the water dipole moment caused by the electric field from the lipids and counter-ions. 


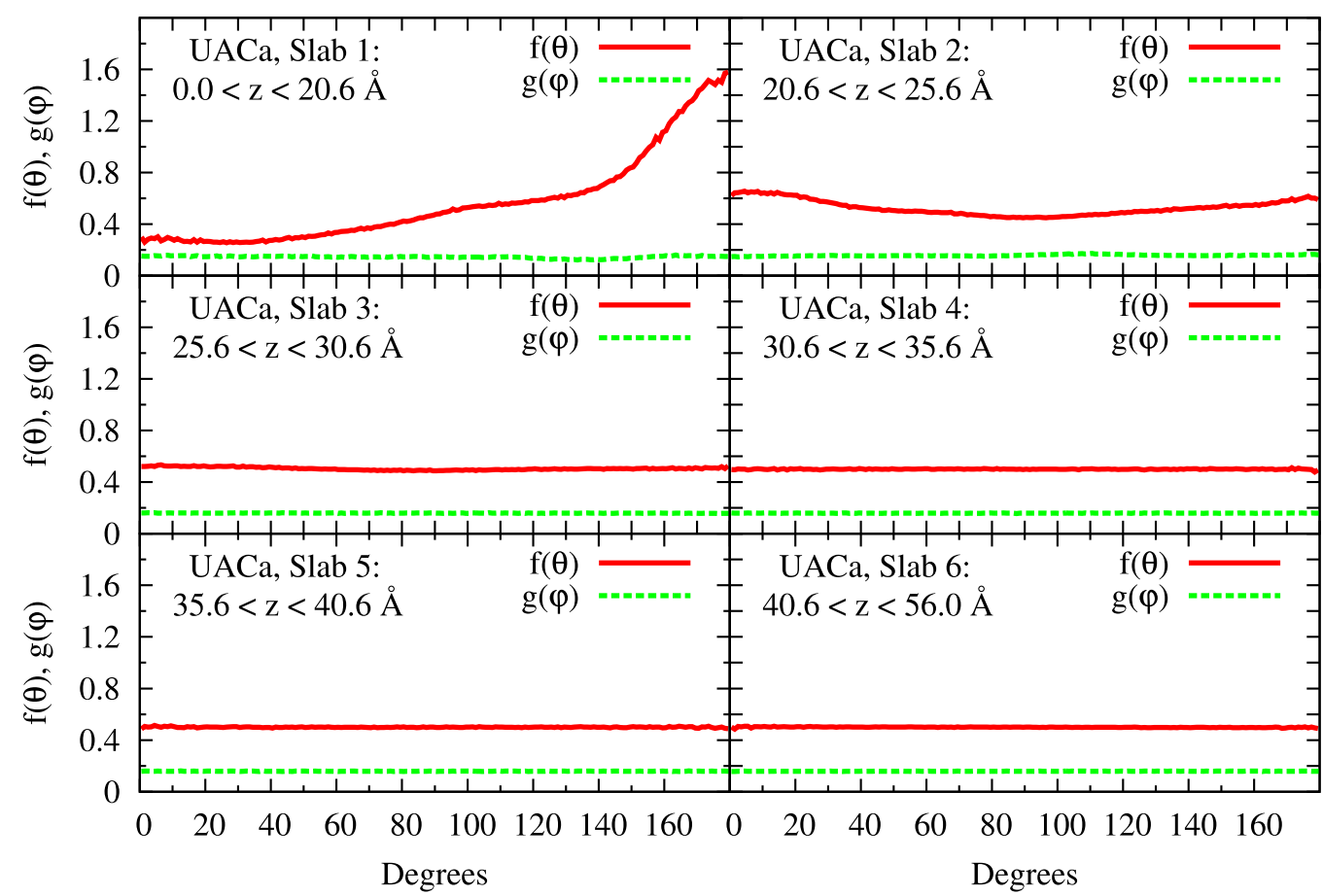

FIG. 13. The polar angle probability density distribution function $f(\theta)$ and azimuthal angle probability density distribution function $g(\phi)$ in six slabs through the DMPG membrane with calcium counter-ions (UACa) at $310 \mathrm{~K}$ and $1 \mathrm{~atm}$. Conditions like mentioned in the caption of Fig. 12.

center in slab 1 with a bimodal distribution in slab 2, which has almost vanished in slab 3. Further out, the orientation is random as in bulk water.

\section{ELECTROSTATIC POTENTIAL}

The electrostatic potential within and outside phospholipid membranes arises because of the alignment of dipolar residues and water dipoles in the region between the aqueous phases and the interior of the bilayer. For charged lipids, like the anionic DMPG, there will also be contributions from the counter-ions. It is experimentally impossible to measure directly the absolute value of the potential in the membrane with the potential outside set to zero. Its value can only be inferred indirectly from the effect it has on some other measurable quantity such as the electrical conductivity. ${ }^{19}$ In the case of phosphatidylcholines of varying chain length and saturation, it has generally been found that the potential determined from electrical measurements on monolayers is of the order of $300-400 \mathrm{meV}^{19}$, whereas the determinations on the basis of ion transport across bilayers and vesicles are generally in the range $200-300 \mathrm{meV} \cdot{ }^{19}$ Various possibilities exist for these differences, but no conclusions have yet been drawn. The estimated potential across the membrane implies an enormous electric field strength within the membrane of $10^{8}-10^{9} \mathrm{~V} \mathrm{~m}^{-1}$. The potential is likely to affect significantly the conformation of translocating membrane proteins and regulating transmembrane transport of molecules and ions as well as enzyme function. An accurate calculation of the potential is therefore necessary and, although progress has been made with indirect methods, the determination of the potential is still quite uncertain and easily at the $\pm 40 \%-50 \%$ level. ${ }^{19}$
We have used our MD simulation results to determine the electrostatic potential as a function of $z$ for both the DMPG and DMPC bilayer membranes. The region between the periodic boundaries along $z$ has been divided into a number of slabs of width $\Delta z$, and the charge density in each slab is determined by counting all charges on atoms in the slab and dividing the result by the volume of the slab. In this way, it is assumed that we may consider the slabs as equipotential surfaces with a non-zero gradient in the potential only along $z$. The random azimuthal orientation of the water dipole moments supports that assumption. The Poisson equation is then reduced to a one-dimensional second-order differential equation of the form

$$
\frac{d^{2} \psi}{d z^{2}}=-\frac{1}{\epsilon_{0}} \sum_{i} q_{i} \rho_{i}(z)
$$

where $\psi$ is the electrostatic potential, $\epsilon_{0}$ is the permittivity of vacuum, and $\rho_{i}(z)$ is the number density of atom $i$ with charge $q_{i}$. The electrostatic potential is determined by integration of Eq. (3) twice. The double integral may be converted into a single integral by an interchange of the order of integration, assuming a length $L$ between the periodic boundaries in the $z$-direction and the center of the membrane at $z=0$. We integrate from $z=-L / 2$ to $z=L / 2$, imposing the periodic boundary conditions $\psi(-L / 2)=\psi(L / 2)=0$, and $\psi(z)$ is given by ${ }^{45}$

$$
\begin{aligned}
\psi(z)= & -\frac{1}{\epsilon_{0}} \int_{-L / 2}^{L / 2} d z^{\prime \prime} \sum_{i} q_{i} \rho_{i}\left(z^{\prime \prime}\right)\left(z-z^{\prime \prime}\right) \\
& +\frac{z+L / 2}{\epsilon_{0} L} \int_{-L / 2}^{L / 2} d z^{\prime \prime} \sum_{i} q_{i} \rho_{i}\left(z^{\prime \prime}\right)\left(L / 2-z^{\prime \prime}\right) .
\end{aligned}
$$




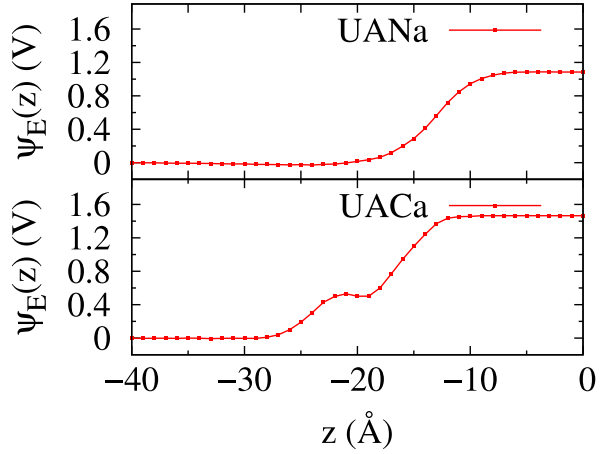

FIG. 14. The electrostatic potential in the DMPG bilayer membrane systems UANa and UACa at $310 \mathrm{~K}$ and $1 \mathrm{~atm}$. The mid-plane of the membrane is at $z=0$.

The results are averaged over 8000 time frames. We tested the sensitivity of the results to the choice of $\Delta z$ and found that they were unchanged with $\Delta z=0.1,0.2$, and $0.5 \AA$. To improve the statistics, we have averaged the results over the two leaflets.

In Fig. 14, we have shown the calculated electrostatic potential for one leaflet. It shows a membrane potential of about $1 \mathrm{~V}$ for the fluid phase of the membrane with the sodium counter-ion (UANa) and a somewhat higher potential of $1.4 \mathrm{~V}$ for the gel phase of the membrane with the calcium counter-ion. The kink in the potential of the UACa system at $z \sim-19 \AA$ reflects the negative charge density in that region as is evident from the charge-density plots in Fig. 15. In the blow-up of the electrostatic potential in Fig. 16, we see that the screening caused by the electric double layer formed by cations and phosphate ions is more effective in the UACa system with the "sharply" defined density profile of the calcium ions compared to the more "diffuse" density profile of the sodium ions (see Fig. 7(b)). Recall that the field outside two plane-parallel plates with opposite charges is zero and is only non-zero between the plates.

For comparison, we have also determined the electrostatic potential in the DMPC bilayer membrane with a choline head group and found a membrane potential of $\sim 500 \mathrm{mV}$, which is rather close to the indirectly determined membrane potentials as discussed above and significantly smaller than in the anionic DMPG bilayer membrane.

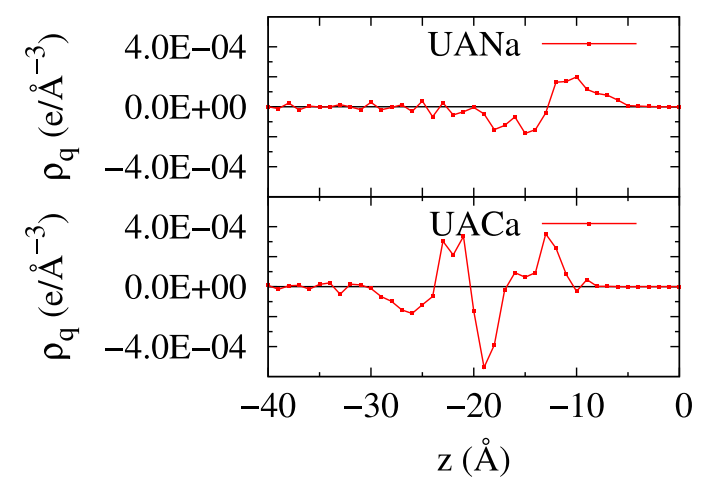

FIG. 15. Charge density along the $z$-direction in the DMPG bilayer membrane systems UANa and UACa leading to the electrostatic potential in Fig. 14. The mid-plane of the membrane is at $z=0$.

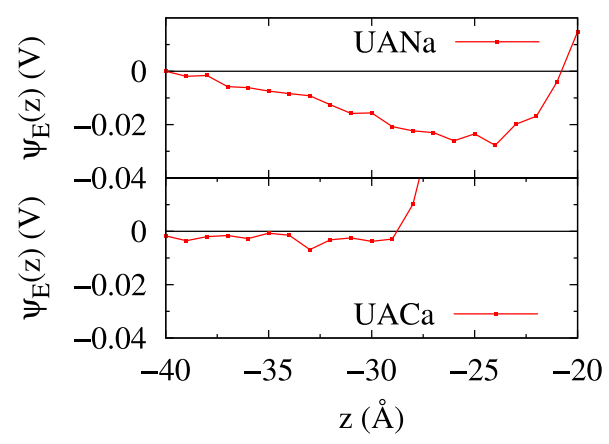

FIG. 16. "Blow up" of the electrostatic potential in the region of the watermembrane interface to highlight the range of the electrostatic potential outside the bilayer membrane systems UANa and UACa. The mid-plane of the membrane is at $z=0$.

\section{CONCLUSIONS}

Experimental studies [Ref. 35] have shown the importance of the counter-ion valency on the phase behavior and hydration characteristics of the anionic DMPG lipid bilayer membrane, consistent with our MD simulation results. We find that at $310 \mathrm{~K}$, the membrane is in the fluid phase with a monovalent counter-ion and in the gel phase with a divalent counter-ion. Our MD simulations suggest that the driving force for the transition from fluid to gel phase is the high affinity of the divalent ion to the phosphate group and its capability to bind simultaneously to two different lipid molecules and thereby create ion bridges between different lipids and greater order in the membrane. This finding is consistent with experiments, ${ }^{34}$ which have shown that the gel-to-fluid transition temperature increases by about $20{ }^{\circ} \mathrm{C}$ when a monovalent counter-ion is replaced by a divalent one.

This phase change is reflected in the diffusion constants for lipids and water molecules in the membrane. In the gel phase, the diffusion constants of lipids and water are about a factor of two smaller than in the fluid phase. There does not seem to be a significant influence of the salt concentration $(0.1 \mathrm{M} \mathrm{NaCl})$ on the diffusion constants of the lipids and water, which are roughly the same as those in the fluid phase with a monovalent counter-ion (UANa). We find that the diffusion constant of water as a function of depth into the fluid DMPG membrane is up to an order of magnitude smaller than in bulk water, just as we found for the fluid phase of DMPC, and that the two membranes have about the same diffusion constant of the lipid molecules in their fluid phases.

On the basis of the water profile, we have determined the number of water molecules required for full hydration of the DMPG membrane in the gel and fluid phases to be 9.7 water molecules per lipid and 17.5 molecules per lipid, respectively. These numbers are smaller than for PC membranes and similar to reported values for PE membranes.

The incoherent scattering functions show that the number of water molecules per lipid moving on the slow time scale of the lipids is $\sim 2.1$ in the gel phase (UACa) and $\sim 4.2$ in the fluid phase (UANa). The larger number in the fluid phase may be related to the presence of more water in it than in the gel phase.

There is reasonable quantitative agreement between the water diffusion constants inferred from the simulation 
at a temperature of $310 \mathrm{~K}$ and from QENS results on samples of single-supported DMPG membranes made at lower temperatures and extrapolated to $310 \mathrm{~K}$. Both indicate the presence of bulk-like water above the membrane and water within the membrane diffusing at a rate up to an order of magnitude more slowly (mean-square-displacement calculations for water molecules in the slabs). At the higher temperature of the simulated QENS spectra, it is impossible to resolve two distinct translational diffusive motions corresponding to bulk-like water and more slowly diffusing water in the membrane interior as is the case in the QENS measurements at lower temperatures. Instead, a weighted average of the diffusion constants in the various slabs is determined from the simulated QENS spectra. The experiments indicate that $\sim 18 \pm 8$ molecules per lipid undergo translational diffusion at the slower rate. However, as discussed above, the sensitivity of the simulations provides evidence of a smaller component of bound water ( $\sim 2$ water molecules per lipid), which diffuses on a time scale comparable to the $\mathrm{H}$ atoms in the lipid molecules. This bound water component is probably too small to be observed experimentally.

For DMPC in the fluid phase, ${ }^{17}$ our simulations gave that 6-7 water molecules/lipid in reasonable agreement with the 7-10 water molecules/lipid derived from experimental quasielastic results.

In both the UANa and UACa systems, we find that there is a preferential orientation of the water dipole moment inside the membrane pointing towards the center of the membrane. The effect is stronger in the UACa system than in the UANa system, a reflection of the different charge distribution in the membranes. The extinction of the electric field outside a double layer is more effective with the divalent counter-ion because of its more confined distribution near the phosphate group as opposed to the monovalent ion whose distribution is more "diffuse" with a tail extending into the bulk water region. Therefore, the electric field extends further out into the bulk water region with the monovalent counter-ion, which also is reflected in the water molecule dipole orientation. Nevertheless, the effect of the electric field from the lipids and counter-ions is limited to a very narrow region outside the membrane.

The membrane potential of a fluid DMPC membrane was found to be $\sim 500 \mathrm{mV}$ in fair agreement with various experimental estimates of $\sim 200-400 \mathrm{mV}$. In comparison, the potentials of the anionic DMPG membranes are somewhat higher: $\sim 1 \mathrm{~V}$ in the fluid state with a monovalent counter-ion and $\sim 1.4 \mathrm{~V}$ in the gel phase with a divalent counter-ion. The transmembrane electric field is thus larger in the anionic membrane than in the zwitterionic neutral membrane, which may be of importance when comparing conformations of translocating membrane proteins and regulation of transport of molecules and ions. There exist no experimental estimates for comparison.

\section{ACKNOWLEDGMENTS}

This work was supported by the U.S. National Science Foundation under Grant No. DGE-1069091. This work utilized facilities supported in part by the National Institute of
Standards and Technology, U.S. Department of Commerce, in providing the neutron research facilities used in this work and facilities supported by the Danish Center for High Performance Computing, DTU, The Technical University of Denmark. A portion of this research at Oak Ridge National Laboratory's Spallation Source was sponsored by the Scientific User Facilities Division, Office of Basic Energy Sciences, U.S. Department of Energy. We thank Zachary Buck, Matthew McCune, and Ioan Kosztin for helpful discussions.

${ }^{1}$ J. Milhaud, Biochim. Biophys. Acta 1663, 19 (2004).

${ }^{2}$ J.-M. Zanotti, M.-C. Bellissent-Funel, and J. Parello, Biophys. J. 76, 2390 (1999).

${ }^{3}$ S. König, E. Sackmann, D. Richter, R. Zorn, C. Carlile, and T. M. Bayerl, J. Chem. Phys. 100, 3307 (1994).

${ }^{4}$ S. K. Pal and A. H. Zewail, Chem. Rev. 104, 2099 (2004).

${ }^{5}$ J. Swenson, F. Kargl, P. Berntsen, and C. Svanberg, J. Chem. Phys. 129, 045101 (2008).

${ }^{6}$ W. Pfeiffer, Th. Henkel, E. Sackmann, W. Knoll, and D. Richter, Europhys. Lett. 8, 201 (1989).

${ }^{7}$ S. König, W. Pfeiffer, T. Bayerl, D. Richter, and E. Sackmann, J. Phys. II 2 , 1589 (1992).

${ }^{8}$ M. C. Rheinstädter, T. Seydel, F. Demmel, and T. Salditt, Phys. Rev. E 71, 061908 (2005).

${ }^{9}$ M. Hishida and K. Tanaka, Phys. Rev. Lett. 106, 158102 (2011).

${ }^{10}$ W. Zhao, D. E. Moilanen, E. E. Fenn, and M. D. Fayer, J. Am. Chem. Soc. 130, 13927 (2008).

${ }^{11}$ A. Miskowiec, Z. N. Buck, M. C. Brown, H. Kaiser, F. Y. Hansen, G. M. King, H. Taub, R. Jiji, J. W. Cooley, M. Tyagi, S. O. Diallo, E. Mamontov, and K. W. Herwig, Europhys. Lett. 107, 28008 (2014).

${ }^{12}$ A. Miskowiec, Z. N. Buck, H. Kaiser, F. Y. Hansen, H. Taub, M. Tyagi, S. O. Diallo, E. Mamontov, and K. W. Herwig, "On the structure and dynamics of water associated with single-supported zwitterionic and anionic membranes" (unpublished).

${ }^{13}$ S. J. Marrink, M. Berkowitz, and H. J. C. Berendsen, Langmuir 9, 3122 (1993).

${ }^{14}$ K. Åman, E. Lindahl, O. Edholm, P. Håkansson, and P. O. Westlund, Biophys. J. 84, 102 (2003).

${ }^{15}$ H. I. Petrache, S. E. Feller, and J. F. Nagle, Biophys. J. 72, 2237 (1997).

${ }^{16}$ Z. Zhang and M. L. Berkowitz, J. Phys. Chem. B 113, 7676 (2009).

${ }^{17}$ F. Y. Hansen, G. H. Peters, H. Taub, and A. Miskowiec, J. Chem. Phys. 137, 204910 (2012).

${ }^{18}$ M. Bai, A. Miskowiec, F. Y. Hansen, H. Taub, T. Jenkins, M. Tyagi, S. O. Diallo, E. Mamontov, K. W. Herwig, and S.-K. Wang, Europhys. Lett. 98, 48006 (2012).

${ }^{19}$ R. J. Clarke, Adv. Colloid Interface Sci. 89-90, 263 (2001).

${ }^{20}$ J. F. Nagle, R. Zhang, S. Tristram-Nagle, H. I. Petrache, and R. M. Suter, Biophys. J. 70, 1419 (1996).

${ }^{21}$ T. T. McIntosh and S. A. Simon, Biochemistry 25, 4948 (1986).

${ }^{22}$ L. Perera, U. Essmann, and M. L. Berkowitz, Langmuir 12, 2625 (1996).

${ }^{23}$ K. A. Riske, H. G. Döbereiner, and M. T. Lamy-Freund, J. Phys. Chem. B 106, 239 (2002).

${ }^{24}$ J. C. Phillips, R. Braun, and K. Schulten, J. Comput. Chem. 26, 1781 (2005).

${ }^{25}$ J. Hénin, W. Shinoda, and M. L. Klein, J. Phys. Chem. B 113, 6958 (2009).

${ }^{26}$ Y. Wu, G. A. Voth, and H. L. Trepper, J. Chem. Phys. 124, 024503 (2006).

${ }^{27}$ J. B. Klauda, N. Kucerca, B. R. Brooks, R. W. Pastor, and J. F. Nagle, Biophys. J. 90, 2796 (2006).

${ }^{28}$ J. Marra, Biophys. J. 50, 815 (1986).

${ }^{29}$ J. Sonne, M. Ø. Jensen, F. Y. Hansen, L. Hemmingsen, and G. H. Peters, Biophys. J. 92, 4157 (2007).

${ }^{30}$ J. B. Klauda, R. M. Venable, J. A. Freites, J. W. O' Connor, D. J. Tobias, C. Mondragon-Ramirez, I. Vorobyov, A. D. MacKerell, Jr., and R. W. Pastor, J. Phys. Chem. B 114, 7830 (2010).

${ }^{31}$ E. Egberts and H. J. C. Berendsen, J. Chem. Phys. 89, 31718 (1988).

${ }^{32}$ E. Egberts, S. J. Marrink, and H. Berendsen, Eur. Biophys. J. 22, 423 (1994).

${ }^{33}$ W.-J. Sun, R. M. Suter, M. A. Knewtson, C. R. Worthington, S. TristramNagle, R. Zhang, and J. F. Nagle, Phys. Rev. E 49, 4665 (1994).

${ }^{34}$ E. Findlay and P. Barton, Biochemistry 17, 2400 (1978). 
${ }^{35}$ P. Garidel, A. Blume, and W. Hübner, Biochim. Biophys. Acta 1466, 245 (2000).

${ }^{36}$ C. Altenbach and J. Seelig, Biochemistry 23, 3913 (1984).

${ }^{37}$ J. F. Nagle and S. Tristram-Nagle, Curr. Opin. Struct. Biol. 10, 474 (2000).

${ }^{38}$ M. Tanaka, G. Girard, R. Davis, A. Peuto, and N. Bignell, Metrologia 38, 301 (2001).

${ }^{39}$ A. D. Enevoldsen, F. Y. Hansen, A. Diama, L. Criswell, and H. Taub, J. Chem. Phys. 126, 194703 (2007).
${ }^{40}$ A. D. Enevoldsen, F. Y. Hansen, A. Diama, H. Taub, R. M. Dimeo, D. A. Neumann, and J. R. D. Copley, J. Chem. Phys. 126, 104704 (2007).

${ }^{41}$ E. Mamontov and K. W. Herwig, Rev. Sci. Instrum. 82, 085109 (2011).

${ }^{42}$ A. Mayer, R. Dimeo, P. Gehring, and D. Neumann, Rev. Sci. Instrum. 74, 2759 (2003).

${ }^{43}$ W. S. Price, H. Ide, and Y. J. Arata, Phys. Chem. A 103, 448 (1999).

${ }^{44}$ J. Teixeira, M.-C. Bellissent-Funel, S. H. Chen, and A. J. Dianoux, Phys. Rev. A 31, 1913 (1985)

${ }^{45}$ J. N. Sachs, P. S. Crozier, and T. B. Wolf, J. Chem. Phys. 121, 10847 (2004). 